\title{
Review Article \\ Carbon Nanotube Electron Sources: From Electron Beams to Energy Conversion and Optophononics
}

\author{
Alireza Nojeh \\ Department of Electrical and Computer Engineering, The University of British Columbia, Vancouver, BC, Canada V6T 1Z4 \\ Correspondence should be addressed to Alireza Nojeh; anojeh@ece.ubc.ca
}

Received 12 August 2013; Accepted 10 September 2013; Published 5 February 2014

Academic Editors: J. Bai, O. Ersen, and G. Zhong

Copyright ( 2014 Alireza Nojeh. This is an open access article distributed under the Creative Commons Attribution License, which permits unrestricted use, distribution, and reproduction in any medium, provided the original work is properly cited.

\begin{abstract}
Carbon nanotubes have a host of properties that make them excellent candidates for electron emitters. A significant amount of research has been conducted on nanotube-based field-emitters over the past two decades, and they have been investigated for devices ranging from flat-panel displays to vacuum tubes and electron microscopes. Other electron emission mechanisms from carbon nanotubes, such as photoemission, secondary emission, and thermionic emission, have also been studied, although to a lesser degree than field-emission. This paper presents an overview of the topic, with emphasis on these less-explored mechanisms, although field-emission is also discussed. We will see that not only is electron emission from nanotubes promising for electronsource applications, but also its study could reveal unusual phenomena and open the door to new devices that are not directly related to electron beams.
\end{abstract}

\section{Introduction}

Electron emission, that is, the transfer of electrons from one medium to another, is ubiquitous in electronics. Electron emission from a material into vacuum (Figure 1) forms the basis of vacuum tubes and amplifiers [1], which predate solid-state devices [2] and still continue to be in widespread use in high-power, high-speed electronics-the mobility of electrons is, after all, very high in vacuum, where they do not face scattering by a lattice. But the applications of electron emission go far beyond signal amplification. From traditional cathode-ray tubes to modern field-emission flatpanel technologies, electron sources can enable bright and fast displays [1]. Electron microscopy has become a popular platform for material and device imaging [3-7], inspection, and failure analysis $[8,9]$, pushing imaging resolution to the sub-ångstrom scale [10]. There have been significant advances in electron emission and control with temporal resolution down to the femtosecond domain and beyond $[7,11,12]$ for time-resolved imaging and diffraction analysis. Analytical characterization techniques such as Auger spectroscopy, electron-energy-loss spectroscopy, and energy-dispersive Xray spectroscopy are now being routinely used and often incorporated into commercial electron microscopes [13].
Electron-beam lithography is a powerful technique for highresolution patterning [14]. In all these systems, the electron emitter (also known as cathode or electron source) is one of the crucial components. Medical imaging is another area where electron sources are in extensive use, notably in Xray generation [1] and electron-beam tomography. Electronbeam welding, machining, and deposition [1] are welldeveloped processes that are widely used in the manufacturing, automotive, aerospace, semiconductor and energy sectors. The removal of nitrogen and sulphur oxides from the emissions of industrial installations can be achieved through electron-beam flue gas treatment [15], toward the reduction of atmospheric pollutants and acid rain. Electron beams can also be used to treat wastewater [16]. In all such systems, the electron source again plays a central role. Electron sources also continue to be an integral part of scientific experiments in accelerators and synchrotrons [1].

An electron source is characterized by a number of key properties such as total emission current, current density, brightness, energy spread, spatial coherence, emission area, lifetime, stability, and noise. Depending on the application, the specific requirements for the electron source could vary significantly. For example, while total current might be more important in electron-beam deposition, brightness 


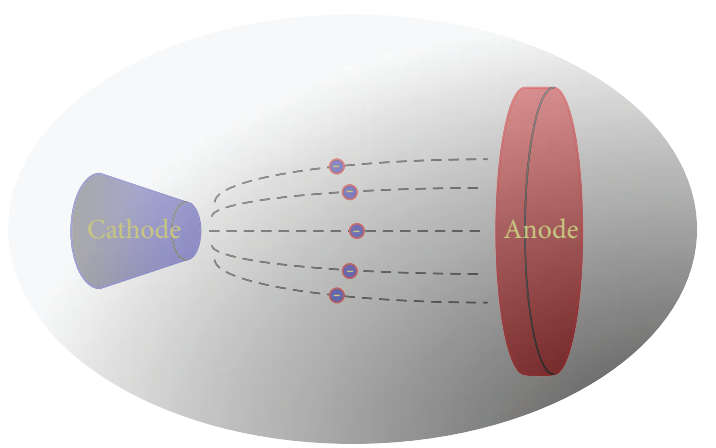

Figure 1: Electrons traveling from cathode (emitter, source) to anode (collector) in vacuum.

and stability could present a greater challenge in highresolution electron microscopy. Today's electron-beam systems have come a long way from their early-20th-century counterparts in terms of electron-optics design, integration, and automation. Similarly, much progress has been made on electron sources; however, remarkably, in many electron-beam devices, the electron source still remains one of the performance bottlenecks. Electron emission is thus an active area of research for scientists and engineers alike.

The advent of nanostructures and, in particular, carbon nanotubes (CNTs) has generated a new wave of interest in electron emission. This paper will explore some of the developments in this area. A large body of literature exists on field-electron emission from carbon nanotubes, spanning almost two decades of work and including several excellent reviews. Other emission mechanisms such as secondary electron emission, photo-electron emission, and thermionic electron emission have been explored only more recently and, so far, to a limited degree compared to field-electron emission. The present paper, therefore, focuses primarily on these less-explored topics. I will start by describing the basics of various electron emission mechanisms and the important parameters to consider and move on to discussing what it is that makes nanostructures so attractive for electron emission. Next, I will present a brief overview of field-electron emission, with emphasis on the difference between carbon nanotubes and bulk emitters in this context. I will then give a more detailed account of particle-assisted electron emission from carbon nanotubes, that is, electron emission as stimulated by other electrons, phonons, or photons. I will then discuss thermionic electron emission from carbon nanotubes, as well as the effect of light in heating nanotubes, the resulting lightinduced thermionic emission and the combined effect of light and heat in electron emission. Next, I will review some of the applications of such electron sources and discuss how the unusual properties of nanotubes enable novel concepts, notably in energy conversion and solar electricity generation. It will be seen that the rich physics of the electron emission phenomena in nanostructures inevitably lead us to new observations and ideas with implications beyond the realm of electron sources per se. I will thus close the paper by proposing a device that I call the optophononic transistor/switch, which has been inspired directly by what we have learnt from light-induced electron emission experiments on carbon nanotubes.

\section{Electron Emission Mechanisms and the Parameters of an Electron Source}

In its simplest form, an electron emitter could be a small metal surface. There exist several mechanisms through which electrons can be emitted from a material into a vacuum. These could involve interactions with fields, waves, or other particles. In thermionic emission [17], the electron source is heated to very high temperatures, typically higher than $1,000^{\circ} \mathrm{C}$. Some of the electrons in the high-energy tail of the Fermi-Dirac distribution thus have sufficient kinetic energy to overcome the material workfunction and escape into vacuum. Heating can be accomplished through various means such as resistive heating or absorption of electromagnetic radiation. Often the electrons will be in thermal exchange with the lattice, in which case one might view phonons as assisting electron emission. This is typically the case when the system is allowed to relax, such as in continuous, constant-current operation. Using fast optical pulses, it is also possible to create a situation where the electrons have a temperature different from that of the lattice, in order, for instance, to study their relaxation dynamics [18]. Fieldelectron emission (field-emission for short) consists of the application of an electric field to the surface of the cathode, with a strength on the order of $\mathrm{V} / \mathrm{nm}$ or higher near the surface, such that the electrostatic potential profile bends down significantly immediately outside the cathode, and the electrons face a thin potential barrier through which they can tunnel quantum mechanically $[19,20]$. Photo-electron emission (photoemission for short) or the photoelectric effect takes place when the absorption of energetic photons by electrons takes the latter to high energy levels, making them overcome the material workfunction and emit into vacuum [21]. This process could include the absorption of a single photon with sufficient energy, or the absorption of multiple photons, the total energy of which would add up to help the electron emit (multiphoton photoemission). Another mechanism through which light may lead to electron emission is optical field-emission [22]. In this scenario, it is suggested that the amplitude of the electric field of the light wave is high to the point that this field can modulate the width of the vacuum barrier sufficiently for a period of time during each cycle of the wave, and electron tunneling (field-emission) can occur. The relative strength of the multiphoton photoemission process and the optical field-emission process can be quantified through the Keldysh parameter [23-26] or the Keldysh parameter together with a scaled light frequency for a wide range of applicability [27]. Electrons can also be ejected from a cathode by absorbing energy from ions or other energetic electrons striking the material. This is called secondary electron emission $[5,6]$, which can be found prominently in scanning electron microscopy and focused ion beam processing. Figure 2 depicts some of the basic emission mechanisms schematically. 


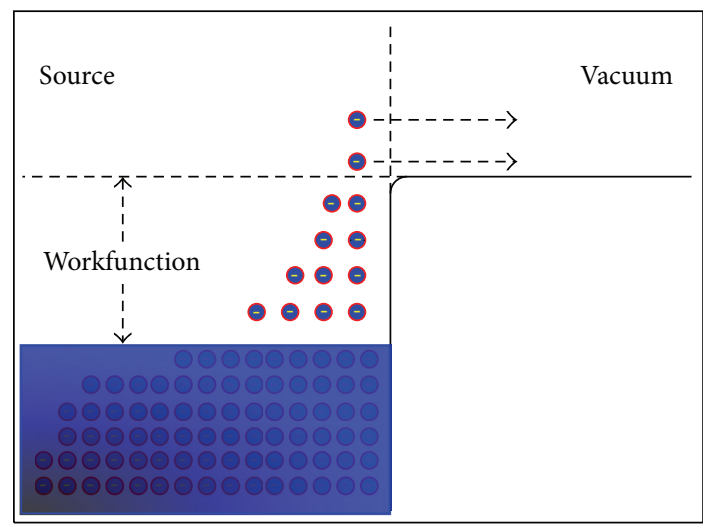

(a)

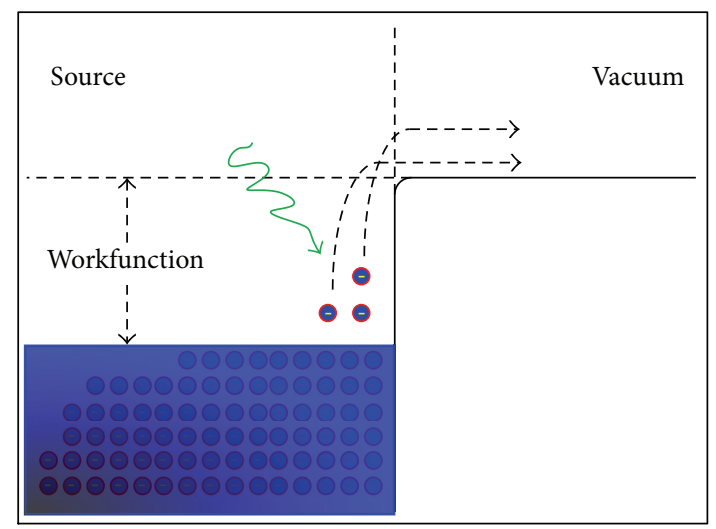

(b)

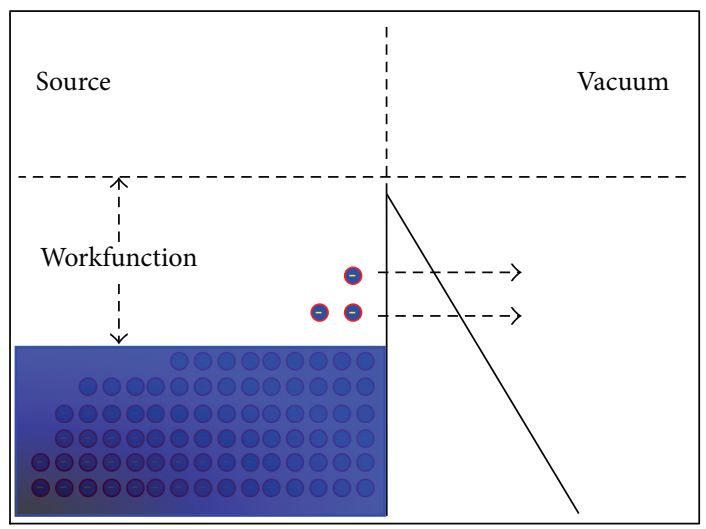

(c)

FIGURE 2: Three common electron emission mechanisms from a metallic source into vacuum: (a) thermionic emission, (b) photoemission, and (c) field-emission.

Combinations of the above are also possible. In Schottky emission [28], the source is heated, but an applied electric field also reduces the height and width of the vacuum barrier. Depending on the relative strength of the two stimuli, one may say that thermally assisted field-emission or fieldassisted thermionic emission is dominant. The former would consist of the tunneling of electrons that are populating higher energy levels because of the elevated temperature, while the latter would involve the electrons overcoming the barrier height, which has been reduced due to the electric field. Schottky sources can combine some of the benefits of thermionic and field-emission sources. Joule heating resulting from the field-emission current itself can also lead to Schottky emission [29]. Similarly, light and applied electrostatic field can have a combined effect: in photo-field-emission, electrons are excited to higher levels through the absorption of light and subsequently tunnel out of the narrower sections of the vacuum barrier [22]. Field-assisted photoemission can also take place when the barrier height reduction by the application of an electric field makes it possible for those electrons photoexcited to higher energy levels to escape, or when a high enough electric field is applied during a photoemission experiment such that the tunneling current is also substantial [30]. The effects of light and heat can also be combined. In photonenhanced thermionic emission, the higher energy levels are populated through the absorption of photons, significantly enhancing thermionic emission [31]. Alternatively, thermally enhanced photoemission could take place, where electrons in the higher-energy regions of the Fermi-Dirac distribution absorb photons, gaining the necessary additional energy to overcome the vacuum barrier. Naturally, light could also be used to heat a cathode and induce thermionic emission. The combined effect of secondary and field-emission is also possible through electron-stimulated field-emission [32,33], where electrons strike the tip of a nanoscale emitter under a strong applied field, leading to highly enhanced emission of further electrons. An applied electric field can also affect the emission and trajectories of secondary electrons emitted from a cathode, enabling voltage-contrast imaging in electron microscopy [34-36]. Finally, it is only natural to expect that more than two stimuli can also be employed, as in combining several of field, light, heat, and primary energetic particles all at once, to induce electron emission.

Various models and theories have been developed for the study of electron emission. Some of the most celebrated ones include the Richardson-Laue-Dushman model of thermionic emission $[37,38]$, the Fowler-Nordheim theory 
of field-emission [20] and subsequent developments [39], the Murphy-Good theory that includes the effects of both heat and field [40], and more advanced treatments including significant recent work by Forbes and colleagues [41-51]; the (generalized) Fowler-DuBridge model that describes the combined effects of heat and multiphoton photoemission [52-54], Spicer's three-step photoemission model $[55,56]$, and Jensen's general formulation that covers thermal-, fieldand photoemission [57]; and models for the analysis of electron scattering in solids (needed in the context of secondary electron emission) such as those by Browning, Joy and colleagues [58, 59].

Depending on the application in mind, several parameters characterize an electron source. The total emission current and the emission current density (current per unit area) are crucial in most cases. The brightness (the emission current density per unit solid angle) and spatial coherence are other important parameters and so is the emission spot size. Sometimes a continuously emitting source is required, while in other cases a pulsed or otherwise time-varying beam is needed, where transient behavior and switching time become critical. Lifetime, stability, and operational environment are other important considerations. Can the source operate only in a clean, high-vacuum environment or is it tolerant of poorer vacuum conditions and, if so, how are its lifetime and stability affected by such nonideal environments? Depending on the emission mechanism, the parameters defining the stimulus are also relevant. For example, for a field-emitter, the necessary electric field to be applied to produce a certain emission current could be of key importance; for a thermionic emitter, the amount of input power required-be it in the form of electricity or light-to reach a certain temperature could be a prime consideration; in a photoemitter, the power, wavelength, polarization, and coherence level of the illuminating light source, as well as quantum efficiency (the ratio of the number of emitted electrons and the number of incident photons), play key parts.

\section{Nanostructures as Electron Sources}

Nanostructures have captured the attention and imagination of researchers in areas as diverse as electronics, optics, mechanics, biomedical technology, and energy, for both fundamental studies and applications. Electron emission, with its crucial role in several of the above areas, has been a popular topic. The rich and still largely unknown physics of nanostructures, primarily due to the strong presence of quantum effects in them, could open the door to novel or enhanced emission mechanisms and characteristics. Conversely, given the richness of the electron emission problem, which involves the solid state, vacuum, and the interface inbetween, electron emission studies could teach us a great deal about nanoscale physics and thus impact other areas; for example, many spectroscopic techniques rely on electron emission as mentioned before. Indeed, the study of electron emission from nanostructures could lead to new discoveries and applications that are themselves not directly related to electron emission. One such example will be discussed later in this paper.

There are also specific and concrete reasons that make nanostructures appealing systems for electron emission. Perhaps in no other nanomaterial are these reasons more prominent than in carbon nanotubes (Figure 3) [60]. A carbon nanotube is made of one or several coaxial tubes composed of carbon atoms in an $\mathrm{SP}^{2}$ bonded, hexagonal lattice. The diameter of these tubes could range from a fraction of a nanometer to tens of nanometers. Depending on the nanotube diameter and chirality (the direction along which an imaginary graphene layer is to be rolled to produce a certain nanotube), a nanotube could be metallic or semiconducting. Carbon nanotubes have a wide array of promising electronic, mechanical, optical, and thermal properties that have put them at the center of attention in the world of nanotechnology for over two decades, with researchers investigating them for nanoelectronics [61], sensing [62], composite materials [63], energy harvesting $[64,65]$, and biomedical technologies [66].

Several of the attributes of nanotubes make them ideal candidates for electron emission devices. These advantages can at first be more readily appreciated in the context of field-emission [67]. A nanotube, with a nanoscale diameter, can have a length that is millions of times larger than its diameter: freestanding nanotubes can be made to lengths of micrometers individually or millimeters $[68,69]$ and beyond in self-supporting arrays (vertically aligned CNT forests). When an electric field is applied to such a high-aspect-ratio structure, it is significantly enhanced near its tip-a fact that we know from basic electrostatics. As a result, one can induce field-emission from carbon nanotubes with an applied electric field on the order of $\mathrm{V} / \mu \mathrm{m}$. This is much smaller than what is needed for field-emission from a flat cathode surface, as the enhancement provided by the nanotube will bring the local field at the tip to the necessary range for electron tunneling. This property is important for low-voltage applications such as portable vacuum electronics and fieldemission displays.

Consider a high-aspect-ratio electron emitter tip carved out of a bulk material. Such a structure will typically have many unterminated atoms and dangling bonds, which could significantly weaken it near the surface. During the rather violent electron emission process and, in particular, when the tip is also heated due to the electron emission current, these loose atoms could move around and even leave the material. This leads to fluctuations in the emission current, as well as a reduction of the emitter lifetime. A carbon nanotube, on the other hand, could have a chemically complete and stable structure; even at its tip, it could be capped with a complete carbon dome (half of a buckyball) or terminated with hydrogen atoms. In addition, the strength of the $\mathrm{SP}^{2}$ carbon-carbon bond gives nanotubes unparalleled mechanical strength. The result is that a higher level of stability, longer lifetime, and operation under poorer vacuum conditions might be expected from carbon nanotube field-emitters.

An individual carbon nanotube can withstand the passage of electric currents up to tens of $\mu \mathrm{A}$. This translates to a maximum current density on the order of $10^{9} \mathrm{~A} / \mathrm{cm}^{2}$, several 


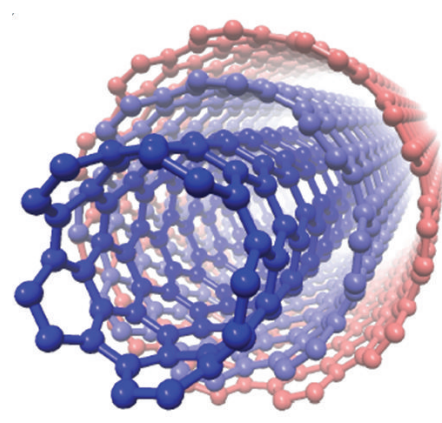

(a)

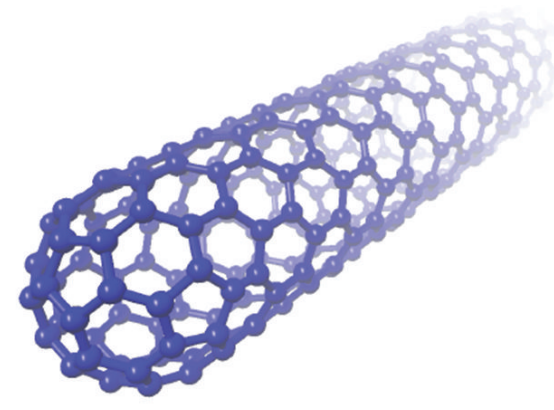

(b)

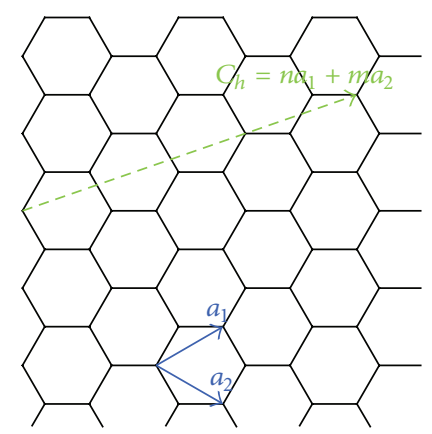

(c)

FIGURE 3: Perspective view of an open-ended multiwalled carbon nanotube (a) and a capped single-walled carbon nanotube (b). (c) shows a graphene lattice and its unit vectors, together with a chiral vector (defined by the indices $n$ and $m$ ) along which the lattice could be rolled to create an $(n, m)$ single-walled carbon nanotube.

orders of magnitude higher than those of the best of conventional conductors. This is enabled by the relatively low resistance of nanotubes to the passage of electrons-meanfree paths on the order of $\mu \mathrm{m}$ have been observed at room temperature [70] — and their mechanical strength and ability to endure high temperatures. Emission current density is itself directly crucial in many cases and also affects the emitter's brightness, which is another critical parameter. Reduced brightness levels of $10^{9} \mathrm{~A} /\left(\mathrm{m}^{2} \cdot \mathrm{Sr} \cdot \mathrm{V}\right)$ have been reported from carbon nanotubes [71], an order of magnitude higher than that of some of the best electron sources in commercial use.

Despite all their benefits, nanotube-based electron emitters also face important challenges. For example, device-todevice reproducibility is still relatively poor-a situation that affects many areas of application: nanotubes with different diameters, chiralities, and cap structures could have significantly different emission properties. With the advantage of having a well-defined nanoscale emission spot also comes the difficulty that the adsorption of one or a few molecules from the environment could affect the emission properties significantly. Similarly, depending on the application, it may be extremely challenging to realize the full benefits of a nanotube emitter: for instance, in an electron microscope or lithography system, one may desire to have the emission spot precisely located on the electron-optical axis. However, this is far from trivial. Another difficulty arises from the fact that the nanotube has to be interfaced with the macroscopic world and the contact point could present a bottleneck to the passage of current. The mechanical strength of the contact point is also critical. Long freestanding nanotubes are also prone to vibration. Nonetheless, most such challenges may be tackled systematically and overcome to satisfactory degrees as needed for various applications.

\section{Field-Emission from Carbon Nanotubes}

Given the obvious suitability of carbon nanotubes for fieldemission, the latter has been by far the most widely studied emission mechanism in them. In addition, a field-emission experiment is fundamentally a simple one to conduct, requiring only a modest power supply and vacuum chamber for basic studies (Figure 4(a)). Field-emission was among the first applications considered for carbon nanotubes, and pioneering studies go back to the early days of nanotube research [72-74]. Field-emission experiments have involved single-walled [75-77], double-walled [78] and multiwalled [79-86] carbon nanotubes and included devices made of individual nanotubes or collections of nanotubes, sometimes mixed with other materials. A large number of the studies have primarily focused on current-voltage characteristics, while others have also investigated the emission spots using field-emission microscopy (Figures 4(b) and 4(c)) [87, 88]. Numerous applications [84] have been demonstrated using nanotube-based field-emitters including flat-panel displays [89-92], electron-beam systems [93-95], mass spectrometers [96], vacuum tubes [84], lighting devices [97], and X-ray imaging tools [98-100], to name but a few. On the theoretical side, studies based on continuum models $[101,102]$ and atomistic simulations [103-111] have been performed, both for the investigation of the electronic structure and for the direct calculation of the emission current $[101,109]$. The effect of adsorbates on the field-emission current from nanotubes has also been studied theoretically $[110,111]$.

The area of field-emission from carbon nanotubes is so vast that it is impossible to do it justice in a section in this paper and, for an in-depth overview, the reader is referred to the excellent book edited by Saito [67] and review articles dedicated to field-emission $[71,112,113]$. However, there is one particular aspect of this topic that I would like to highlight. Many works in the literature have used the Fowler-Nordheim model to fit measurement data from carbon nanotube fieldemitters. While this is an important model and widely useful, one has to keep in mind that it was originally developed for a metallic emitter with a flat surface. These conditions are not necessarily satisfied by carbon nanotube-based emitters and, as a result, this model may not be appropriate for studying field-emission from nanotubes under all circumstances. This issue has too often been overlooked in the literature, sometimes with negative consequences for the understanding of the experimental results. For example, despite the clear deviation of the measured data from a straight line on the 


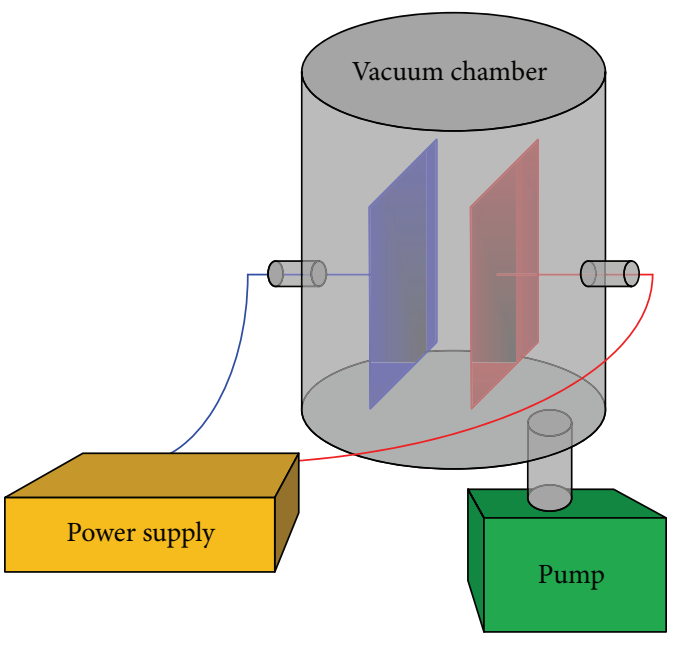

(a)

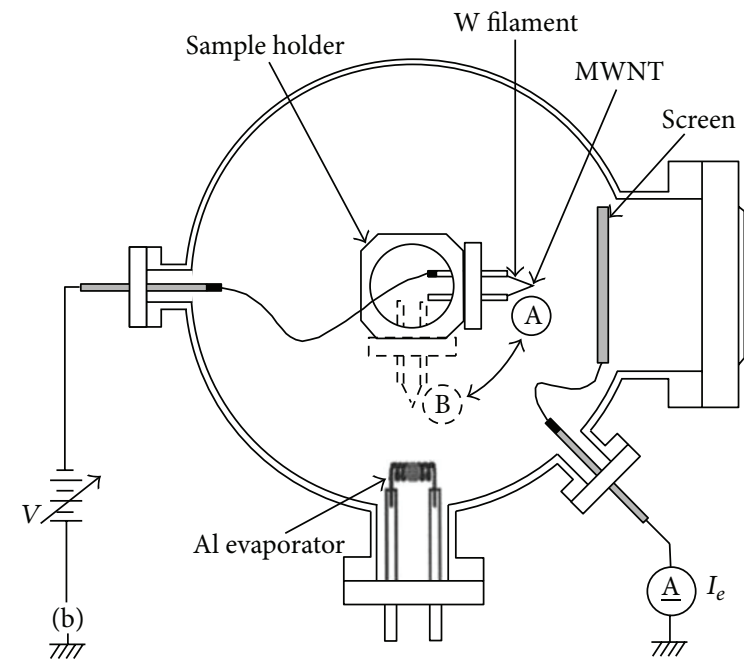

(b)

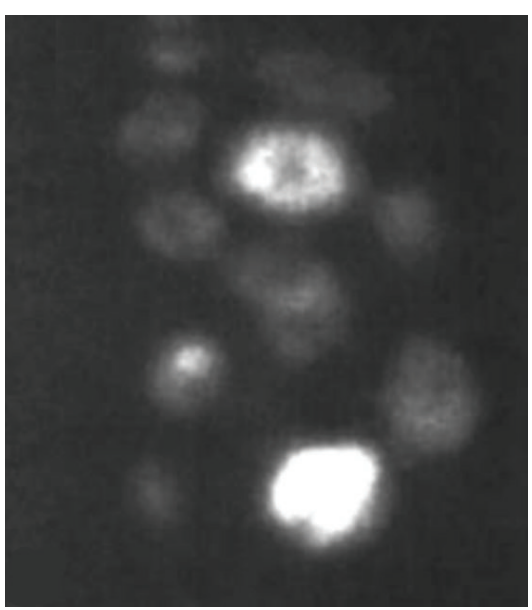

(c)

FIgURE 4: (a) A field-emission setup: only a simple vacuum chamber and power supply are needed for basic studies; (b) a more advanced apparatus, equipped with aluminum coating capability and an imaging screen for field-emission microscopy (FEM) of carbon nanotubes; (c) emission pattern from a multiwalled carbon nanotube recorded using the apparatus shown in (b) ((b) and (c) reprinted with permission from [88]. Copyright 2010, American Vacuum Society).

so-called Fowler-Nordheim plots in many cases, forcing a straight-line fit has sometimes been practiced, leading to unreasonably high estimates of the field enhancement factor. Fortunately, this has not always been the case. Several works such as $[77,114,115]$ have pointed out the deviation from linear Fowler-Nordheim behavior in field-emission experiments on nanotubes. Such deviations have been explained through cooperative effects among the tips in an emitter consisting of a collection of nanotubes [116], the difference in the energy band structure of nanotubes and conventional emitters [117], field penetration and induced apex dipoles [118], the nonlinear nature of the tunneling potential barrier as well as its angle dependence $[119,120]$, variation of the local field [121], and the slowing down of the rate of reduction of the barrier width/height as the applied field increases [109].

\section{Particle-Assisted Electron Emission from Carbon Nanotubes}

5.1. Secondary Emission from Carbon Nanotubes. When a beam of energetic electrons strikes a solid, the impinging electrons collide with the nuclei, electrons, and quasiparticles (such as plasmons) inside the material and are scattered. Many such scattering events take place and the "primary" electrons follow a random-walk path deep into the material, until they lose all of their kinetic energy due to the scattering events. The trajectories of all these electrons, which can be simulated using a Monte Carlo approach [122], when combined, typically form a teardrop shape (Figure 5(a)). This is the interaction volume of the material with the primary electron beam. When the collisions are with other electrons, the latter could gain enough kinetic energy to exit 


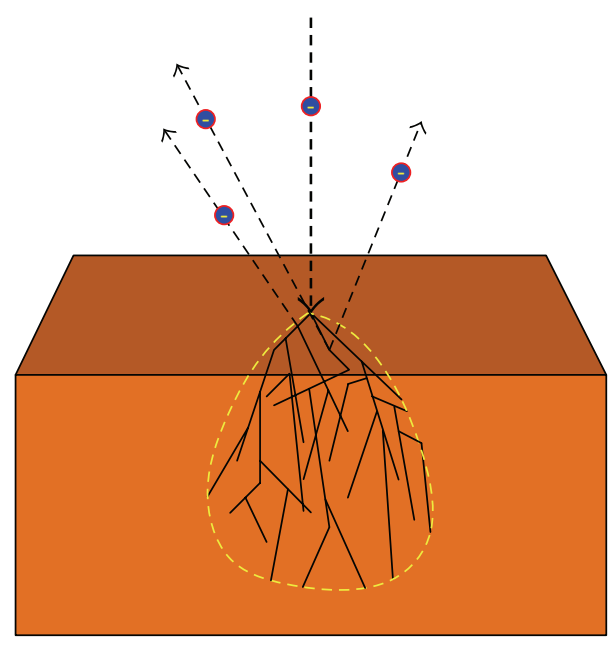

(a)

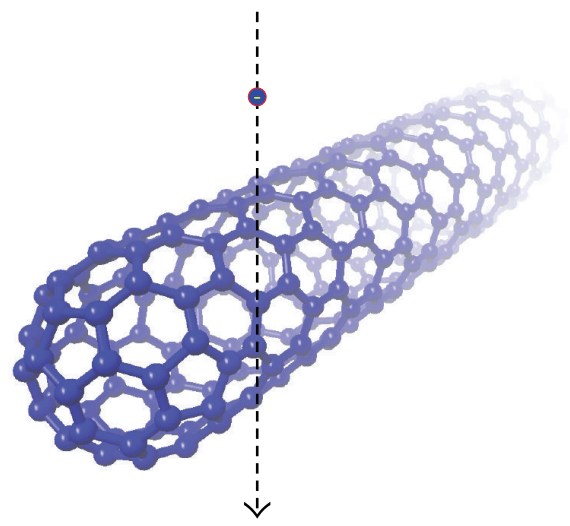

(b)

FIGURE 5: (a) Scattering of energetic primary electrons incident on a bulk solid, and the resulting secondary electrons exiting the surface; (b) in a carbon nanotube, due to the low interaction volume, a low level of scattering of the primary electrons is expected.

the material (in essence, an ionization process could take place). By convention, those electrons being ejected from the material with kinetic energies of less than $50 \mathrm{eV}$ are called secondary electrons (note that, in a general sense, secondary electrons could also be produced by ion irradiation). These typically exit the sample from depths of less than a few tens of nanometers and so contain information about the surface. Secondary electrons are thus used for surface image formation in scanning electron microscopy (SEM).

Let us now consider a carbon nanotube being bombarded by an energetic primary electron beam, perpendicular to its axis. Given the hollow structure and nanoscale diameter of the material, it hardly presents any appreciable interaction volume with the primary beam (Figure 5(b)), in contrast to the situation in a bulk. Therefore, one would expect little scattering, if any, to take place and, as a result, a very low secondary electron emission yield. Yet, surprisingly, carbon nanotubes are readily visible in the scanning electron microscope, often with good contrast. Of course, image formation and contrast mechanisms in an SEM are complex phenomena, influenced by artifacts such as charging and contamination $[5,6]$. Certainly, charging and voltage contrast appear to play an important role in scanning electron microscopy of carbon nanotubes [123-125]. Electron-beaminduced current has also been reported to play a part [126].

The fundamental question of whether a carbon nanotube can produce a substantial number of secondary electrons is an important and interesting one. Indeed, it has been proposed that direct interaction with the primary beam should lead to appreciable, although modest, secondary electron emission $[127,128]$. On the other hand, an abnormally high secondary electron emission yield has also been reported for carbon nanotubes [129]. Our own experiments have revealed that nanotubes have a low secondary electron yield, consistent with the conclusions of $[127,128]$. It appears that the high yield reported in [129] is an artifact of the interpretation of the experiments, as we have detailed in a comment [130]. Nonetheless, even the modest secondary electron yield of $<1$ that we have measured is somewhat surprising, given the small interaction volume of the nanotubes with the primary beam.

Secondary electron emission from carbon nanotubes has also been studied theoretically. Using first-principles quantum mechanical simulations, we have shown that placing an external electron inside the hollow structure of a nanotube can have a dramatic effect on the electrostatic potential landscape, shifting the nanotube energy levels up considerably and facilitating the emission of the electrons occupying those levels [128, 131]. Using a discrete-energy-loss Monte Carlo approach, we have simulated electron trajectories, electron energy loss, and secondary electron emission from nanotubes [132], obtaining secondary emission yield values in good agreement with our experimental measurements, reconfirming that secondary electron emission from nanotubes is weak $[127,133]$.

The interaction of primary electron beams with carbon nanotubes has also been studied extensively using the dielectric response function, with focus on inelastic scattering of electrons with primary energies of less than $30 \mathrm{keV}$, which are those typically relevant to SEM $[134,135]$. The findings have been used in a Monte Carlo framework to simulate electron transport and energy dissipation in collections of multiwalled carbon nanotubes [136]. Analytic expressions for electron/proton inelastic scattering and energy loss in carbon nanotubes have also been reported [137]. Monte Carlo modeling has also been used to study electron trajectories and backscattering in aligned carbon nanotube arrays (CNT forests) $[138,139]$ : we have observed that, due to their mostly empty volume, CNT forests allow for an unusually high electron penetration range. The effect of different scattering models on electron penetration and backscattering studies in nanotubes has also been investigated [140] by implementing the various models in a Monte Carlo code [141].

Aside from regular secondary and backscattered electron emission, nanotubes allow for uncommon phenomena to occur. We have demonstrated that, if a nanotube placed on a dielectric surface is biased just below the threshold of field-emission and its tip irradiated with a primary electron, the extra energy provided by this primary electron can 


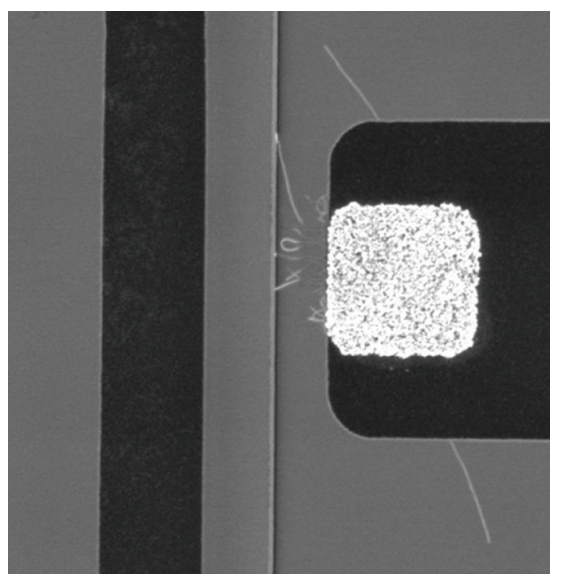

(a)

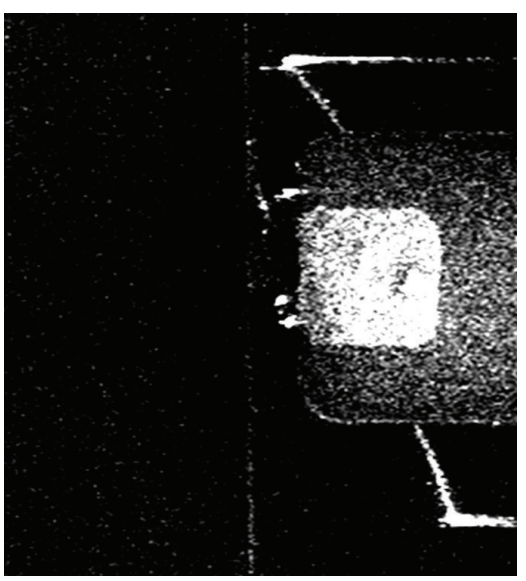

(b)

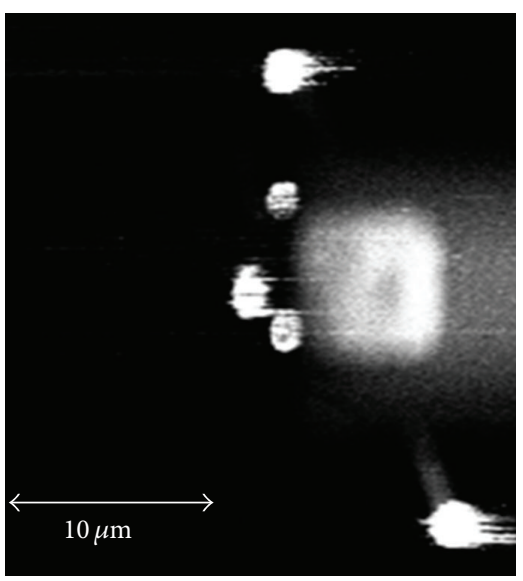

(c)

FIGURE 6: Electron stimulated field-emission from carbon nanotubes being imaged in an SEM: (a) several single-walled carbon nanotubes resting on a silicon dioxide surface and connected to an electrode on the right side. The white square is the catalyst island used for nanotube growth; (b) upon the application of an electric field near the onset of field-emission, the nanotube tips appear as bright spots, indicating the emission of a large number of electrons every time the primary beam of the microscope scans past those points; (c) the tips become brighter under slightly higher applied electric field (reprinted with permission from [32]. Copyright 2004, AIP Publishing LLC).

trigger the emission of a large number of electrons from the nanotube tip, with an electron gain of $>2,000$ [142]. We call this phenomenon electron stimulated field-emission $[32,33]$. If an array of nanotubes is used, electron gains of up to 19,000 can be obtained [143]. Given the high gain, these devices effectively act as a nanoscale current-controlled vacuum transistor. When these experiments are performed in an SEM, since a high number of electrons are emitted and collected by the secondary electron detector every time the primary beam scans past the nanotube tip, the tip appears as an extremely bright spot on the image (Figure 6) [32]. This method may thus provide a convenient way of visualizing the emission spots of other nanoscale emitters as well.

Secondary electrons can also be emitted as a result of irradiation of nanotubes with ions. Studies of the effect of electronic excitations in ion collisions and energy loss distribution of proton beams incident on nanotubes [144-146] are important in this context. One consideration in experiments involving the interaction of energetic electron or ion beams with nanotubes is potential damage and atom dislocation. These effects could also be exploited in a beneficial way for engineering and tailoring the structure of nanotubes. For a review on this topic, the reader is referred to [147].

\subsection{Phonon-Assisted Electron Emission from Carbon Nan-} otubes. Other particles can also induce electron emission from carbon nanotubes. As mentioned before, in the case of thermionic emission, if the electrons are at thermal equilibrium with the lattice, that is, if electrons and phonons are in a state of ongoing energy exchange, one may view the electron emission phenomenon as being at least partially assisted by phonons. On the other hand, the effect of phonons on electron emission could potentially be more direct: in experiments on electron emission from the side surface of a nanotube biased through electrodes on the two ends, it was observed that electrons "overflowed" from the surface, which was explained by the electrons' absorption of hot optical phonons [148].

5.3. Photoemission from Carbon Nanotubes. The workfunction of carbon nanotubes is generally understood to be in the $4-5 \mathrm{eV}$ range $[149,150]$, although values outside this range have also been reported. For the photoelectric effect to take place, therefore, photons with energies greater than this value are needed. This is in the ultraviolet (UV) or X-ray ranges. Photoemission from carbon nanotubes using high-energy photons has been studied by many, primarily in the form of spectroscopy for the investigation of the electronic structure of nanotubes under various conditions [151-156] and photoemission microscopy $[157,158]$. The photon energies in these cases are high enough to probe deep electronic levels. However, photoemission experiments using photon energies very near the nanotube workfunction, for electron emission from the Fermi level or the conduction band, as is the case in photocathode applications, have been relatively scarce and started much later compared to field-emission studies. As well, these have often involved a collection of nanotubes, rather than individual nanotubes.

In experiments involving the illumination of a nanotube film using pulsed laser beams, it was observed that the wavelengths of $532 \mathrm{~nm}$ and $355 \mathrm{~nm}$ (corresponding to photon energies of $2.33 \mathrm{eV}$ and $3.49 \mathrm{eV}$, resp., both below the workfunction) could only heat the nanotubes and induce thermally assisted field-emission, whereas the wavelength of $266 \mathrm{~nm}$ (corresponding to a photon energy of $4.66 \mathrm{eV}$ ) led to an additional, field-assisted photoemission regime [159]. In experiments on arrays of aligned, millimeter-long multiwalled nanotubes with macroscopic lateral dimensions, we observed that a continuous-wave laser with a wavelength of $266 \mathrm{~nm}$ illuminating the nanotube forest (Figure 7) can 


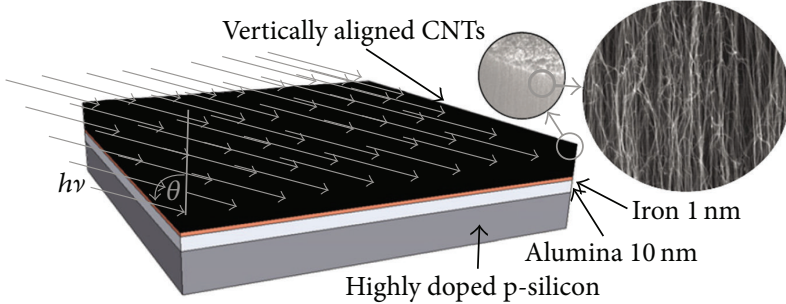

(a)

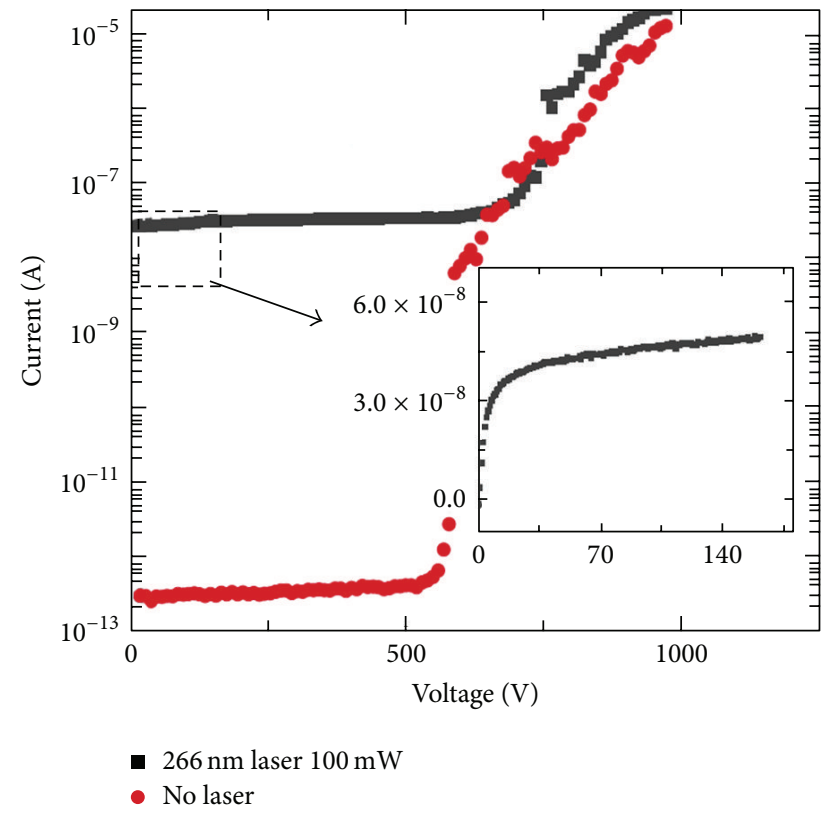

(b)

Figure 7: (a) A forest of vertically aligned carbon nanotubes, grown through chemical vapor deposition on a silicon substrate using an iron catalyst layer on an alumina buffer layer, is illuminated by ultraviolet light in photoemission experiments. Inset shows a scanning electron micrograph of the side of the forest, revealing the overall alignment of the nanotubes; (b) the electron emission current of the device as a function of cathode-anode voltage with and without illumination (reprinted with permission from [160]. Copyright 2010, AIP Publishing LLC).

induce photoemission with a quantum efficiency of $\sim 10^{-5}$; increasing the applied collection voltage to go beyond the purely photoemission regime led to a quantum efficiency of $\sim 10^{-3}[160]$. These values are $2-4$ orders of magnitude higher than those reported for randomly distributed nanotubes and are quite remarkable for a metallic system. Another finding was that, when the incident light was polarized to have its electric field parallel to the axis of the nanotubes, it was absorbed much more efficiently and was thus expected to lead to a higher electron emission current. For perpendicular polarization, the light penetrated much deeper into the nanotube forest before being entirely absorbed. An interesting observation was made in experiments with a similar light source but on a sparse collection of individual nanotubes resting on a silicon dioxide surface and connected to metallic electrodes on one end [161]: we noticed that the high level of photoemission current measured could not be explained based on the laser intensity, surface area of the nanotubes, and a reasonable value for quantum efficiency. It appeared that a strong enhancing mechanism should be at play, possibly based on optical antenna effects, leading to an overall higher level of optical absorption than expected [161].

Indeed, photoemission from nanostructures represents an interesting regime of operation. Photoemission, itself, is a quantum mechanical phenomenon, and can be explained by considering the particle nature of light; in traditional photoemission experiments on bulk solids, the wave nature of light may not be directly manifest. However, considering that typically the dimensions of nanostructures are comparable with the wavelength of light in the UV-visible-IR (infrared) range, wave phenomena such as diffraction, interference, and antenna effects could be prominent in nanostructures [162166]. Consequently, photoemission from them could reveal a rich combination of effects, where both the wave nature and the particle nature of light are strongly manifest. A similar situation exists in photodetectors and light emitters based on carbon nanotubes. This combination and the design space that it opens up allow for engineering novel devices and imagining new applications. For example, we have discussed the possibility of creating micro/nanoscale networks for onchip wireless interconnect at optical frequencies $[167,168]$. A closely related topic is the use of plasmons to enhance photoemission properties [169-174].

When a strong electric field is applied to carbon nanotubes under photon irradiation, the resulting field-emission current could dominate over the photoemission current. If the field strength is lower than the levels required for pure field-emission, the combined effects of light and fieldthe so-called photo-field-emission effect-may be observed. When we illuminated carbon nanotube forests with a visible laser $(532 \mathrm{~nm}$ in wavelength, corresponding to a photon energy of $2.33 \mathrm{eV}$ ), we observed a range of applied electric fields where the emission current could be explained by photo-field-emission [175]. In that range, the field-emission current matched the theoretical prediction using a value for effective workfunction equal to the nanotube workfunction minus the photon energy. This suggests that photon absorption may lead to a substantial number of electrons populating energy levels higher than the Fermi level by the photon energy, and subsequently tunneling under the influence of the applied electric field.

\section{Thermionic Emission from Carbon Nanotubes}

The strong carbon-carbon bond in nanotubes, the mechanical strength it provides, and the resulting fact that nanotubes can withstand high temperatures make them promising candidates for thermionic electron emission sources. On the other hand, their low resistance to the flow of electrons suggests that it may not be trivial to generate enough heat in them using resistive heating. Moreover, given their high thermal conductivity, one expects that any heat generated in a region of a nanotube should spread out significantly, making 
it difficult for it to reach the high temperatures needed for thermionic emission, given its relatively high workfunction. Nonetheless, in practice the situation could be different from this picture in major ways. For example, structural defects in a nanotube could lead to higher levels of electron scattering and enable resistive heating. In fact, heating of an individual nanotube and thermionic emission have been reported [176]. Resistive heating and thermionic emission from nanotube bundles, yarns, and sheets have also been demonstrated [177179]. These experiments also allowed the estimation of the nanotube workfunction. During field-emission, Joule heating due to the emission current can itself lead to substantial heating of the nanotube [180]. Such a behavior has also been reported in [29] and shown to result in Schottky electron emission.

6.1. Light-Induced Thermionic Emission from Carbon Nanotubes. A cathode could be heated to thermionic emission temperatures through illumination by electromagnetic waves such as visible or IR light. However, because of the good thermal conductivity of typical cathode materials, they act as heat sinks: the heat generated at the illuminated spot will spread into a wide area outside the spot. Therefore, it is difficult to heat the cathode appreciably, unless a large amount of optical energy is used. This can be achieved using the high intensities available from powerful pulsed lasers, and the resulting heated area will be much larger than the illuminated spot. The high-intensity light source requirement and the challenges associated with the need for thermal management and prevention of heat spread to the rest of the device or system make these cathodes complex and expensive. Carbon nanotubes are understood to have high or at least moderate thermal conductivities [181-186], to the point of being proposed for applications in thermal management [187]. One thus expects that, similar to the situation in other optically heated thermionic cathodes, the light-induced heating of a nanotube forest should be challenging. Surprisingly, this is not the case and CNT forests can be heated efficiently with extremely low optical powers.

Consider the experiment depicted in Figures 8(a) and 8(b). A carbon nanotube forest with macroscopic dimensions is illuminated on one side using a laser beam. The beam diameter is on the order of tens to hundreds of micrometers, substantially smaller than the lateral dimensions and height of the forest, all of which are on the order of millimeters. Since the thermal conductivity of nanotubes is high along their length (the vertical direction), it is expected that the generated heat diffuses along this direction, heating an entire stripe from the top to the bottom of the forest. However, we have observed that, quite counterintuitively, the heat can remain essentially localized to the illuminated spot [188]. Figure 8(c) shows the resulting incandescence from the hot spot. (Note that the laser light has been blocked by a filter in front of the camera taking this photo, and only the incandescence is seen.) We have called this unusual effect "Heat Trap" and explained it as follows.

First of all, one has to consider the fact that, in all thermal conductors, lattice thermal conductivity is itself a function of temperature $(T)$ : as temperature increases to hundreds of degrees Celsius and beyond, thermal conductivity typically drops as $1 / T$. This is due to the nature of the phonon scattering events. In nanotubes, a steeper drop, including a second-order temperature dependence term in the denominator, has been observed [189-191] and attributed to umklapp and second-order three-phonon scattering [189, 192, 193]. Imagine that we start by illuminating a spot on the sidewall of the nanotube forest with a very low optical intensity. The resulting heat will dissipate easily through the length of the nanotubes, and the temperature will not rise noticeably. If we gradually increase the intensity, at some point a threshold is reached where the rate of heat generation will be slightly higher than that of heat dissipation, and the temperature will rise by a small amount. With this increase will come a decrease in thermal conductivity, which will in turn reduce the amount of heat transfer out of the illuminated spot and lead to a further increase in the local temperature. The system will thus enter a positive feedback cycle that rapidly drives down the thermal conductivity and increases the temperature. Under this condition, the heated area effectively acts as a thermal insulator and accumulates the generated heat, hence the name Heat Trap [188]. The result is that, due to the low heat loss to the surroundings, a small amount of optical power is sufficient to heat the illuminated region to extremely high temperatures and induce thermionic emission. Note that Heat Trap can also happen when the top surface of the nanotube forest (where the nanotube tips are exposed) is illuminated. This may be understood more easily since, in this case, the heat transfer in the transverse direction (perpendicular to nanotube axes) is obviously low as it relies on inter-nanotube thermal conductivity, rather than conductivity along the length of the nanotubes.

A natural question is whether a similar effect would not also be expected in regular bulk materials, as their thermal conductivity, too, drops with temperature. The answer is that, in principle, it could be, but given the slower negative dependence of thermal conductivity on temperature in these materials, the optical intensity required to reach the Heat Trap threshold would be expected to be much higher, and the resulting hot spot would not be as localized to the illuminated spot as is the case in nanotube forests. We believe this is the reason why this effect has not been reported in bulk materials. (However, it is conceivable that this phenomenon could be produced in other nanostructures.) There is also another significant difference between a nanotube forest and a typical bulk conductor. Consider the power equilibrium equation at the illuminated spot under steady-state conditions.

Input optical power $=$ power lost due to incandescence + power lost due to thermionic electron emission + power lost due to heat dissipation to the surroundings.

In the case of an isotropic bulk, heat dissipation occurs uniformly in all directions into the volume. In the case of a nanotube forest, on the other hand, where thermal conductivity is much higher along the nanotube axes than in the perpendicular direction, heat dissipation happens highly anisotropically, essentially in one dimension (Figure 9), and the last term of the above equation takes a different form than in an isotropic bulk. This effect also contributes to the 


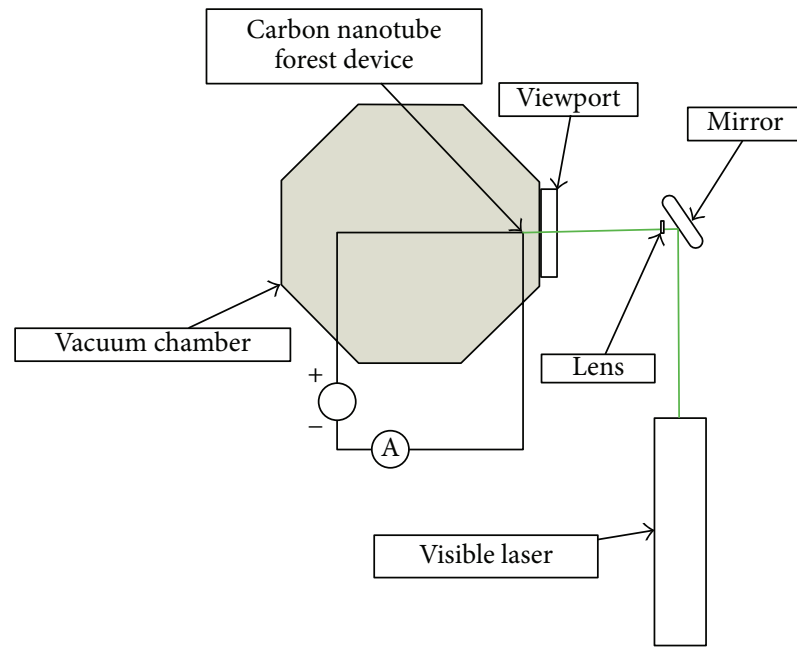

(a)

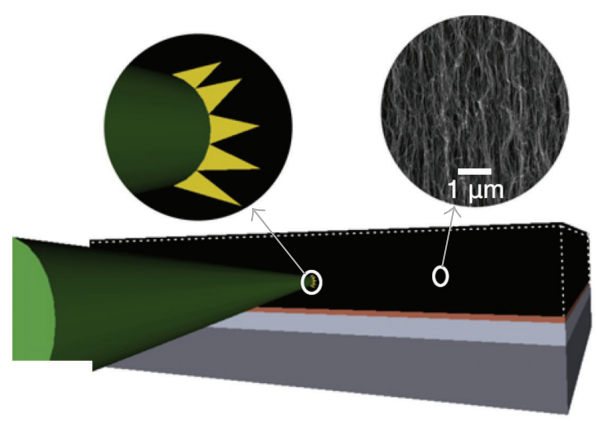

(b)

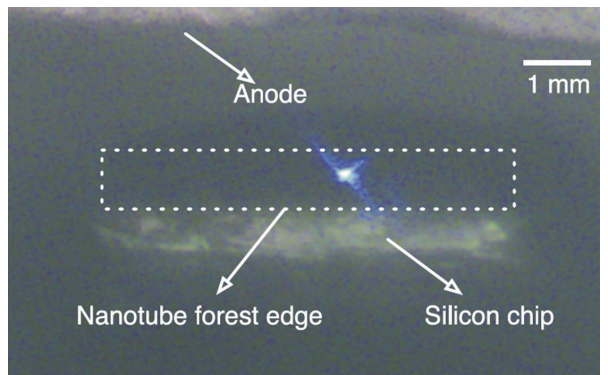

(c)

FIGURE 8: Laser-induced localized heating of the side of a carbon nanotube forest through the Heat Trap effect: (a) schematic of the experimental apparatus; (b) device diagram showing the focused nature of the illumination. Note that the illuminated spot size is significantly smaller than the lateral dimensions of the carbon nanotube forest, which are on the order of millimeters; (c) photo of the nanotube forest (highlighted by the dashed box) showing the incandescence glow from the locally heated spot. The photo was taken while a filter in front of the camera blocked the laser light, so that only the incandescence of the hot spot is seen (reprinted from [188]. Copyright 2011, with permission from Elsevier).

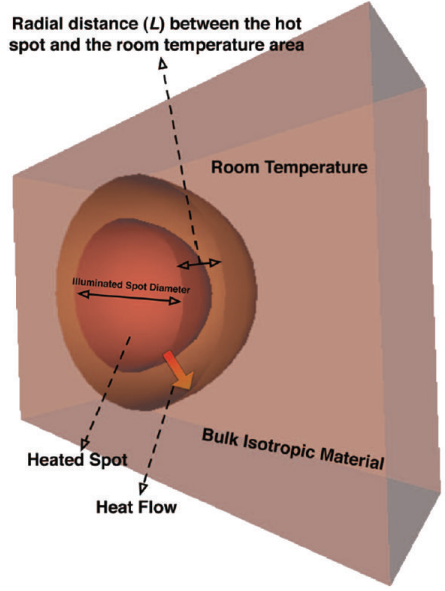

(a)

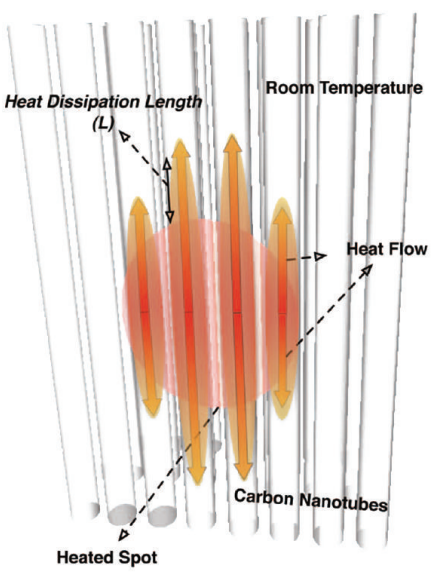

(b)

FIGURE 9: Comparison between heat dissipation out of an illuminated spot in an isotropic bulk conductor (a) and a carbon nanotube forest (b) (reprinted from [194]). 


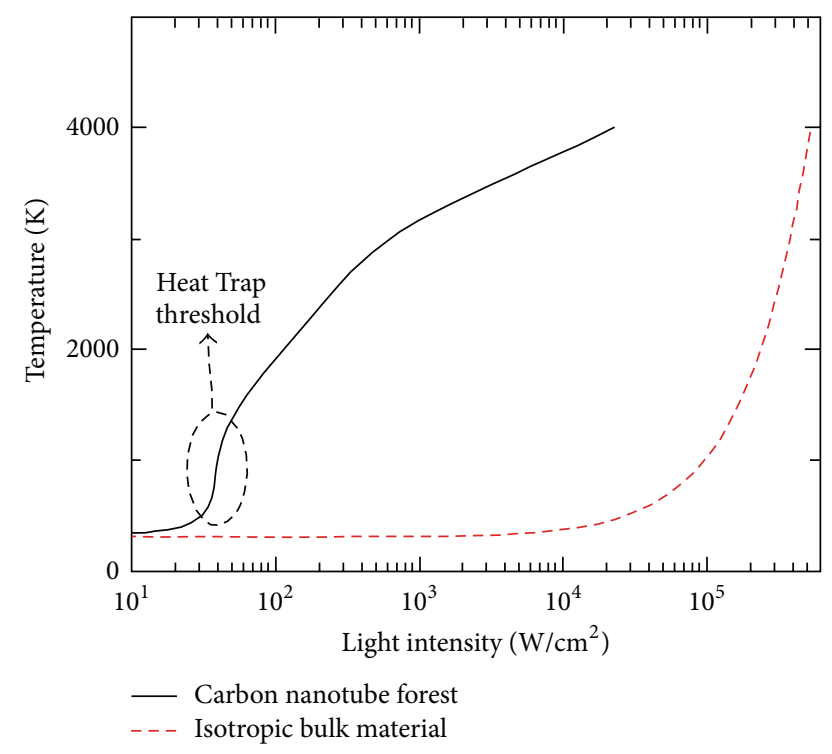

FIGURE 10: The temperature of an illuminated spot as a function of the intensity of incident light for a carbon nanotube forest and a regular isotropic bulk conductor; the intensity required for significant heating in the nanotube forest is over three orders of magnitude smaller than that in the bulk. This is due to the Heat Trap mechanism (reprinted from [194] with modifications).

Heat Trap threshold intensity being much lower in nanotube forests.

Figure 10 shows simulation results for a comparison between the nanotube forest and an isotropic bulk in terms of the temperature attained versus illumination intensity, where a steep temperature rise can be seen in the nanotube forest at an intensity that is several orders of magnitude lower than that required for any appreciable heating in the bulk conductor [194]. In comparative experiments, we have observed that, under intensities on the order of $\sim 50 \mathrm{~W} / \mathrm{cm}^{2}$, which are sufficient for Heat Trap and the heating of nanotube forests to a few thousand degrees, a tungsten surface is not heated to any noticeable level [188]. In the case of the nanotube forest, we have measured temperature gradients as high as $1,200 \mathrm{~K} / \mathrm{mm}$ between the illuminated region and the remainder of the structure [195].

We have also studied the dependence of this localized heating effect on the wavelength and polarization of the incident beam of light. The response is relatively independent of the wavelength in a broad range of UV-visible-IR: when illuminating the CNT forest with laser beams with wavelengths of $405 \mathrm{~nm}, 514.5 \mathrm{~nm}, 658 \mathrm{~nm}$, and $1064 \mathrm{~nm}$, similar levels of electron emission currents were obtained for similar levels of illumination intensity in all cases [196]. It was also observed that the effect of combining beams with different wavelengths is essentially the same as that of using a single wavelength with intensity equal to the sum of those of all the individual beams. This broad spectral response has important practical implications as shall be discussed later but is not surprising as carbon is known to be a good absorber of electromagnetic radiation. In particular, nanotube forests have been shown to be extremely black in a wide range of wavelengths [197, 198].

We have found that the thermionic emission current is sensitive to the polarization of the incident beam of light: when the electric field vector of the light beam is aligned parallel to the axis of the nanotubes in the forest, the measured emission current is significantly higher than when the field vector is perpendicular to the nanotubes [196]. This may be explained based on the optical absorption depth in the forest in both cases: simulations show that, for parallel polarization, the entire optical power is absorbed within a few tens of nanometers of the sidewall surface, while for perpendicular polarization the absorption is much weaker and the beam can penetrate several micrometers into the forest [196]. As a result, the electrons emitted in the former case are closer to the surface and can escape the material more efficiently, hence the higher emission current.

\subsection{Photoemission, Thermionic Emission, and Nonlinear} Photoemission from Carbon Nanotubes. The combination of light and heat in inducing electron emission from materials has been of interest since the first half of the last century as exemplified by the Fowler-DuBridge theory [5254]. Typically, a photoemission experiment can be augmented by adding a substrate heater to allow for the study of the effect of temperature on the photoemission current, as the light source itself may not be intense enough to lead to substantial temperature rise. For example, photo- and thermionic emission have been reported from potassiumintercalated arrays of carbon nanotubes using such a setup [199]. On the other hand, in some cases, intense pulsed lasers are used, which can heat the source to thermionic emission temperatures, but typically these are of higher wavelengths (such as IR) and their photon energies are not sufficient to induce the photoelectric effect, unless several photons are absorbed by a single electron to cause multiphoton photoemission. With carbon nanotube forests, we have an interesting regime of operation due to the Heat Trap effect that enables their localized heating with a low-power beam of light, even that of a continuous-wave UV laser with a maximum power of only a few hundred milliwatts. Indeed, as we illuminated the forest with this laser beam, at very low intensities (before the Heat Trap threshold), we observed a linear photoemission (one-photon photoemission) behavior. As the intensity was increased, a second-order nonlinear regime was observed [200] that fit the two-photon photoemission term of the generalized Fowler-DuBridge theory quite well. Eventually, by increasing the intensity further, the temperature rose to the point that thermionic emission took over. The resulting current-temperature graph experimentally confirms the behavior predicted by the FowlerDuBridge theory in a very broad range of temperatures. The exploitation of such combined effects of light and heat in a cathode has been proposed for solar energy conversion [31].

6.3. Applications of Light-Induced Thermionic Emission from Carbon Nanotubes. Heat Trap allows the nanotube forest 
to reach thermionic emission temperatures with input optical intensities as low as a few mW. This opens up new possibilities. In addition, given the localized nature of the hot spot, there is no concern about damage to the rest of the cathode structure, and hence there is no need for additional thermal isolation that can add weight, complexity, and cost. As such, this electron source may enable simple, compact/miniaturized, and inexpensive vacuum electronic devices; it could also be useful in electron-beam systems.

Consider the particular example of electron-beam lithography. Although it is versatile and capable of extremely high patterning resolution, its usage is still limited in the semiconductor fabrication industry because of its low throughput, which primarily results from its "direct write" nature. Among the approaches to improve the performance of electron-beam lithography is the usage of shaped [201-203] and multiple [204-211] beams. For example, rather than using a focused beam with a nanoscale spot size to create an entire pattern point by point in a full-direct-right strategy, one may employ a wide beam shaped with various apertures to project large portions of the pattern with simple shapes such as rectangles and triangles. A fine scanning beam could subsequently complete the patterns in corners, edges, and other areas where higher patterning flexibility is needed. Such a strategy would be beneficial especially in cases where many repetitions of simple shapes are needed, such as in integrated circuits. The usage of multiple beams in parallel (Figure 11) could also increase the throughput. This could be achieved by having the different beams write different sections of the same chip, or each a different chip, all simultaneously. Shaped- and multiple-electron-beam lithography have been pursued by major industrial players such as IBM, KLA-Tencor, MAPPER Lithography, and IMS Nanofabrication. In order to shape the electron beam, one may, for instance, use shaped apertures. For multiple parallel beams, the use of multiple electron sources, multiple apertures with addressable blanking capability to turn individual beams on and off on demand, and arrays of addressable reflectors have been considered. Most of these approaches are based on manipulating the electron beams directly and only after the electrons have been emitted.

Alternatively, it is possible to use photocathodes, where the electron beams are controlled by the corresponding optical beams from outside the vacuum chamber. This could afford the system additional simplicity and flexibility. For example, one may use the mature, flexible, and programmable technologies of shaping optical beams (think of today's projectors) to emit electron beams with corresponding shapes from a photocathode. Similarly, an array of addressable, focused optical beams could create multiple parallel electron beams. Photocathodes, on the other hand, face challenges of low quantum efficiency or ultrahigh vacuum requirements. It would thus be desirable to accomplish a similar task using optically induced thermionic emission. However, in regular bulk cathodes, as discussed, extremely high optical intensities would be needed for each beam, rendering a multi-beam configuration impractical. More importantly, the fundamental property of heat spread to a wide area makes it impossible to create a shaped electron beam from a shaped optical beam, or multiple closely packed electron beams

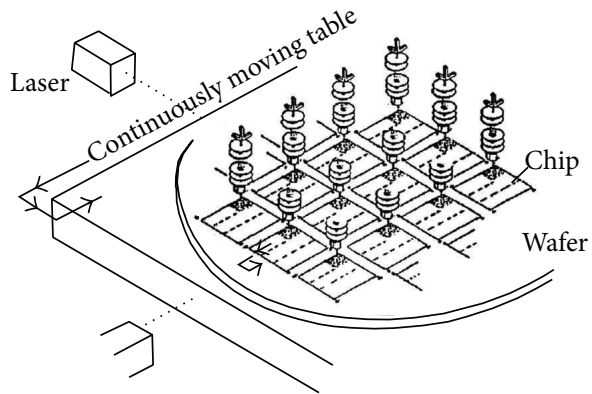

FIGURE 11: Diagram of a multiple-electron-beam lithography tool (reprinted with permission from [206]. Copyright 1996, American Vacuum Society).

from multiple optical beams. Here again, the localized lightinduced heating of carbon nanotube forests through Heat Trap may offer a solution. We have demonstrated the creation of multiple electron beams with spot sizes comparable to those of the exciting optical beams, and electron beams with a linear (rather than circular) cross section, all from a single thermionic cathode - the carbon nanotube forest [212].

This localized heating effect also has implications beyond the realm of conventional electron beam technologies. Note how the heated region in this case acts as a poor thermal conductor, retaining the heat locally and maintaining a high temperature gradient, in excess of $1,000 \mathrm{~K} / \mathrm{mm}$ [195]. At the same time, the material seems to retain reasonable electrical conductivity, as suggested by the fact that electron emission occurs from the hot spot. For efficient thermoelectric power generation, one needs a material with good electrical conductivity and poor thermal conductivity in order to obtain a good figure of merit. This has been the key goal in thermoelectric research for a long time, but this combination has proven to be difficult to achieve. The opposite situation-that of good thermal conductivity and poor electrical conductivityis more readily available, such as in diamond. Some of the research in the area of thermoelectrics thus focuses on materials with structures that could suppress phonon transport. Our explanation of the Heat Trap mechanism relies on the rapid drop of lattice thermal conductivity with temperature. It, therefore, implies that, at high temperatures, a type of phonon "traffic jam" might be taking place, reducing thermal conductivity. As a result, it seems that Heat Trap might offer possibilities in efficient thermoelectric conversion of light to electricity.

A close cousin of thermoelectric power generation is thermionic power generation. The basic idea is simple and dates back to the early 20th century [213-217]. Consider the apparatus depicted in Figure 4(a), including a cathode and an anode in vacuum. If the cathode is heated to thermionic emission temperatures, some of the emitted electrons will be collected by the anode and fed to an external circuit, even if a voltage bias is not applied (or a reverse bias is applied). In principle, cathode heating could be achieved by a source of light, such as sunlight, the device acting as a thermionic solar converter. This structure would naturally use a broad portion of the solar spectrum, which is a significant advantage over 


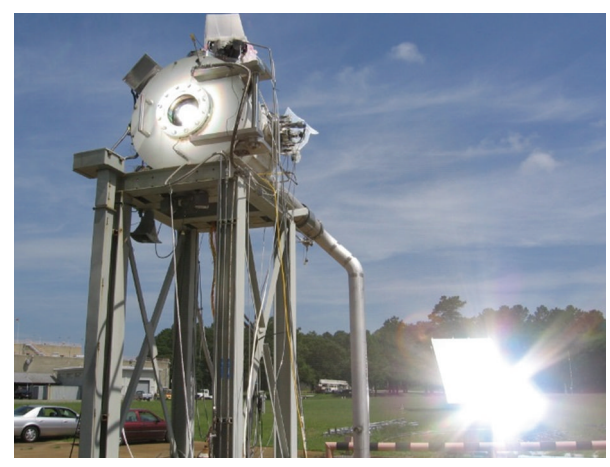

(a)

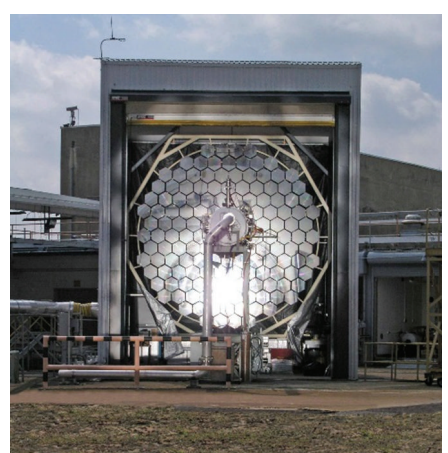

(b)

FIgURE 12: Solar thermionic convertor at the NASA Marshall Space Flight Center facility: (a) shows the convertor chamber, as well as the heliostat in the distance; (b) shows the view from the other side of the convertor chamber, where the concentrator array located behind can be seen. Note the size of the structure compared to the vehicles in the background (lower left corner of (a)) (reprinted with permission from [219]. Copyright 2006, AIP Publishing LLC. High-resolution images courtesy of Steven F. Adams).

photovoltaics. In addition, given the exponential dependence of current on temperature in thermionic emission, a small improvement in focusing the sunlight, which would presumably lead to a temperature increase in the cathode, could lead to a large increase in the emission current and conversion efficiency. Indeed, conversion efficiencies greater than 50\% have been predicted [218]. The device can also deliver high power density and current density, which are both important from the points of view of cost and portability. In addition, given the lack of sensitive crystal materials, it can be robust to radiation.

The above characteristics make thermionic electricity convertors attractive for solar electricity generation. Interest in solar thermionics became strong starting over half a century ago, and there has been particular interest for space power applications, especially given the prospects for high output power per unit volume or mass. For example, NASA has had several programs on solar thermionic conversion, including the Solar Energy Thermionics (SET) program and the High Power Advanced Low Mass (HPALM) program, and demonstrated conversion efficiencies of around $7 \%$ as early as in the 1960s [219]. However, there have been significant challenges, including those having to do with the heating of the cathode: as discussed, very high optical intensities are required to heat a bulk metal to thermionic emission temperatures. This can be appreciated by the fact that large light collection and focusing equipment have been required (Figure 12), which are typically available only in specialized facilities such as NASA's Jet Propulsion Laboratory Table Mountain facility or the Marshall Space Flight Centre [219]. Even so, in many of the tests performed, the cathode has been heated using electrical heating, rather than actual heating by sunlight. Moreover, the heat spread to the rest of the structure creates practical problems and necessitates the use of heat isolation and cooling strategies, which add to the complexity, size, and cost of the device.

Given that low optical intensities can induce thermionic emission from carbon nanotubes and the heat remains contained within the illuminated spot, the Heat Trap mechanism could provide a solution to the above challenges. On average, the sunlight intensity is $0.1 \mathrm{Wcm}^{-2}$ on the surface of the Earth. Given the typical Heat Trap threshold intensity of $\sim 50 \mathrm{Wcm}^{-2}$, a focusing factor of 500 in area should be sufficient for solar thermionic emission from nanotubes. This could be achieved easily using an off-the-shelf glass lens. Indeed, we have demonstrated solar thermionic emission using a simple configuration (Figure 13(a) compared with Figure 12) [194]. If a positive voltage is applied to the anode relative to the cathode, the device acts as a solar electron source. If a negative voltage is applied to the anode, the electrons have to overcome the resulting potential barrier using their own kinetic energy, thus delivering power to the system. Figure 13(b) shows both regimes of operation. In the absence of an external voltage and by connecting the anode and cathode through a resistive load, the device delivers power to the load. For example, our prototype generated $1.3 \mathrm{~V}$ across a $10 \mathrm{M} \Omega$ resistor. Although the efficiency of this early prototype was low, its power density and current density were already comparable to those of state-of-the-art photovoltaic convertors [194]. Moreover, there is significant room for improving the efficiency due to the exponential nature of thermionic emission. A promising approach to efficiency increase consists of tipping the balance between energy loss through incandescence and electron emission, toward the latter. For this, it is desirable to operate at lower temperatures to reduce black body radiation; however, in order to achieve a high electron emission current at lower temperatures, the material workfunction must be reduced, which may be accomplished using, for example, surface adsorbates or structural modifications.

Another crucial issue in thermionic conversion is the so-called space charge effect: the Coulomb repulsion of the already-emitted electrons hinders further electron emission from the cathode. This poses a serious limit on the ultimate achievable efficiency, and mitigation strategies consist of using a plasma environment (rather than vacuum), where the positive charge of the gas ions balances the negative charge of the electrons, as well as using very small anode-cathode 


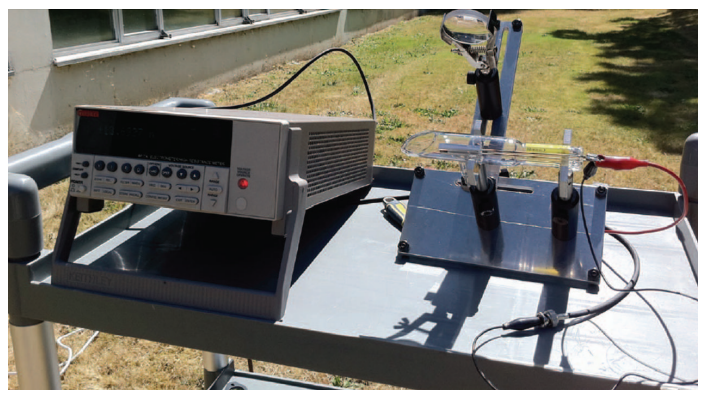

(a)

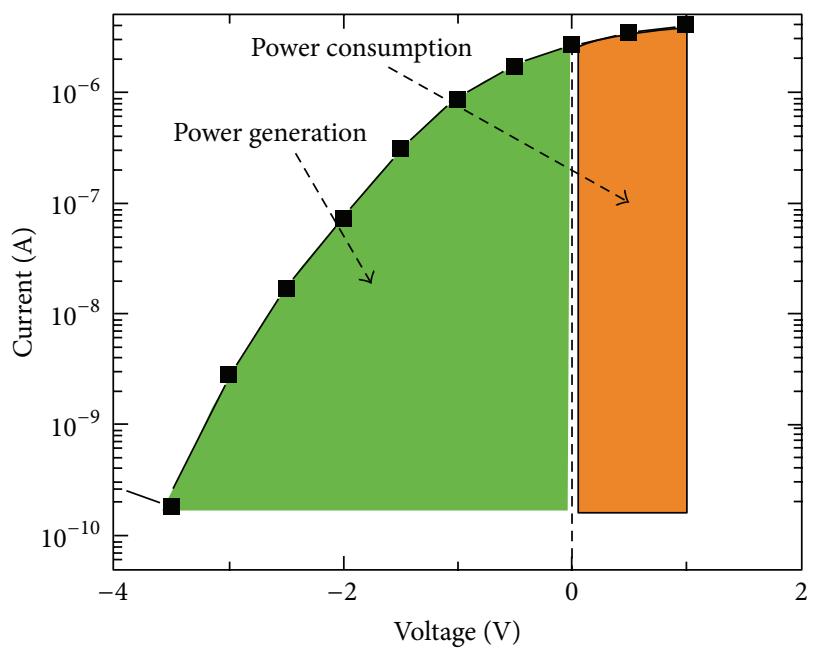

(b)

FiguRE 13: (a) Solar electron source based on a carbon nanotube forest in a portable glass vacuum chamber under test on a sunny day in Vancouver, BC. A glass lens approximately $5 \mathrm{~cm}$ in diameter is sufficient for inducing solar thermionic emission. Compare the size and simplicity of this device with the structure shown in Figure 12; (b) current-voltage characteristics of a nanotube-based thermionic convertor, showing both the power consumption and the power generation regimes of operation (reprinted from [194]).

gaps [218] to improve electron collection and reduce the time the electrons spend in the gap. More elaborate engineering of the gap region is also possible through the use of additional electrodes to apply electric or magnetic fields and modify the emitted electron distribution so as to minimize space charge effects [220].

\section{Beyond Electron Sources: What Else Do We Learn from Carbon Nanotube Electron Emitters?}

Given the rich nature of the electron emission problem and the fact that there are still many aspects of it that we do not fully understand, it is only logical that the study of carbon nanotube electron emitters should have implications in other application areas as well. Here I will propose an idea that has naturally developed from the Heat Trap effect discussed above.

Phononics consists of the use of phonons (rather than electrons) as carriers of information or the control of phonons toward other goals such as thermal management. For example, if one could use phononic devices to control, direct, and utilize the heat generated in electronic circuits to add processing power, one would achieve an improvement over the present situation, where we face the major problem of removing the heat from the circuit, only to send it to waste. Directional thermal effects were first observed many years ago, but the past decade has seen several advances in the field of phononics, with models for thermal diodes $[221,222]$ and experimental realizations of micro/nanoscopic solid-state thermal rectifiers [223, 224]. Researchers have also proposed thermal transistors, gates, and memory [225-227]. There have also been significant efforts on phononic crystals [228].

A compelling idea would be to combine optical signals with phononic devices, in a manner similar to the way optics and electronics have combined to form the field of optoelectronics. Consider a simple structure such as the one shown in Figure 14. The idea is to use a gate signal to control the flow of phonons from the source to the drain electrode (the word electrode might be appropriately replaced by "phonode"), similar conceptually to the manner in which an electric fieldeffect transistor operates. The current in this case would consist of phonon flow, and the voltages would be replaced by the temperatures on the source and drain. Instead of 


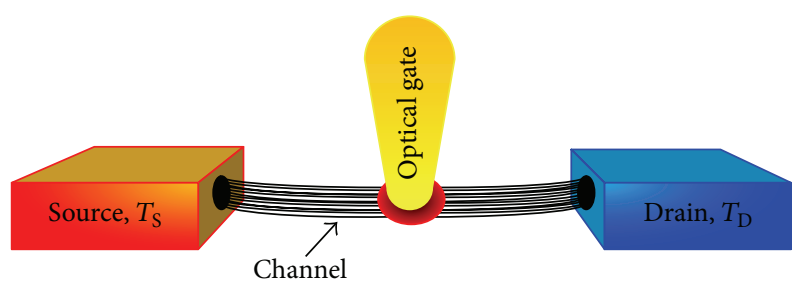

Figure 14: Proposed structure for an optophononic transistor/switch: when the gate signal (light beam) is off, a temperature difference between the source and drain leads to phonon flow through the channel (carbon nanotube bundle); when the light beam is on with intensity above the Heat Trap threshold, a section in the middle of the nanotube bundle heats to high temperatures, leading to a local decrease in thermal conductivity and reduced phonon flow. Modulating the light intensity around the Heat Trap threshold would lead to the modulation of the phonon flow between the source and the drain.

the gate voltage, an optical signal would be used, similar to a phototransistor. Assume that the channel is made of a bundle of parallel carbon nanotubes. Without a gate signal, the channel would have good thermal conductivity and, if the source temperature is higher than the drain temperature, a net phonon flow from the source to the drain would result. The higher the temperature difference between the source and the drain, the higher the rate of this flow would be. Now, if we illuminate the gate region with a beam of light with intensity around the Heat Trap threshold, we would be operating in a regime where the thermal conductivity of the channel is a strong, nonlinear function of the light intensity (recall the positive feedback cycle in Heat Trap). Therefore, the phonon flow between the source and drain could be modulated strongly using small variations in the intensity of the incident optical beam. We could call this an optophononic transistor/switch (one might also be compelled to use the name thermophotonic). This concept is to be distinguished from acoustooptics, optophonics [229] or photoacoustics [230], although such phenomena might also take place in the proposed device and influence its behaviour.

\section{Summary}

The goal of this paper was to present an overview of the area of electron emission from carbon nanotubes, not only covering the much-studied topic of field-emission, but also emphasizing some of the less-explored emission mechanisms including secondary emission, photoemission, and thermionic emission. I attempted to highlight the fact that nanotube electron emitters are such rich physical systems that their study could lead to findings with ramifications beyond the traditional realm of electron-source devices. I hope the paper can help spark further interest in the area.

\section{Conflict of Interests}

The authors declare that there is no conflict of interests regarding the publication of this paper.

\section{Acknowledgments}

The author thanks the University of British Columbia, the Natural Sciences and Engineering Research Council of Canada, the Canada Foundation for Innovation, the British Columbia Knowledge Development Fund, the BCFRST Foundation, and the British Columbia Innovation Council for financial support of his group's work on electron emission, some of which has been reviewed in this paper.

\section{References}

[1] J. A. Eichmeier and M. K. Thumm, Eds., Vacuum Electronics, Components and Devices, Springer, Berlin, Germany, 2008.

[2] B. G. Streetman, Solid State Electronic Devices, Prentice Hall, Englewood Cliffs, NJ, USA, 1990.

[3] J. J. Bozzola and L. D. Russell, ElectronMicroscopy, Jones and Bartlett, London, UK, 1999.

[4] S. L. Flegler, J. W. Heckman Jr., and K. L. Klomparens, Scanning and Transmission Electron Microscopy: An Introduction, Oxford University Press, Oxford, UK, 1993.

[5] L. Reimer, Image Formation in Low-Voltage Scanning Electron Microscopy, vol. 12 of Tutorial Texts in Optical Engineering, SPIE Optical Engineering Press, 1993.

[6] L. Reimer and H. Kohl, Transmission Electron Microscopy: Physics of Image Formation, Springer Science+Business Media, New York, NY, USA, 2008.

[7] A. H. Zewail and J. M. Thomas, 4D Electron Microscopy, Imaging in Space and Time, Imperial College Press, London, UK, 2010.

[8] P. Sandland, W. D. Meisburger, D. J. Clark et al., "An electronbeam inspection system for X-ray mask production," Journal of Vacuum Science \& Technology B, vol. 9, p. 3005, 1991.

[9] D. Meisburger, U. S. Patent 5,502,306, 1996.

[10] P. E. Batson, N. Dellby, and O. L. Krivanek, "Sub-ångstrom resolution using aberration corrected electron optics," Nature, vol. 418, no. 6898, pp. 617-620, 2002.

[11] M. Gao, C. Lu, H. Jean-Ruel et al., "Mapping molecular motions leading to charge delocalization with ultrabright electrons," Nature, vol. 496, pp. 343-346, 2013.

[12] M. Kruger, M. Schenk, and P. Hommelhoff, "Attosecond control of electrons emitted from a nanoscale metal tip," Nature, vol. 475, pp. 78-81, 2011.

[13] G. Botton, "Analytical electron microscopy," in Science of Microscopy, P. W. Hawkes and J. C. H. Spence, Eds., Springer, 2007.

[14] K. Suzuki and B. W. Smith, Eds., Microlithography, Science and Technology, CRC Press, London, UK, 2007.

[15] A. G. Chmielewski, E. Iller, Z. Zimek, M. Romanowski, and K. Koperski, "Industrial demonstration plant for electron beam flue gas treatment," Radiation Physics and Chemistry, vol. 46, pp. 1063-1066, 1995.

[16] J. G. Trump, E. W. Merrill, and K. A. Wright, "Disinfection of sewage wastewater and sludge by electron treatment," Radiation Physics and Chemistry, vol. 24, no. 1, pp. 55-66, 1984.

[17] S. Dushman, “Thermionic emission," Reviews of Modern Physics, vol. 2, no. 4, pp. 381-476, 1930.

[18] X. Y. Wang, D. M. Riffe, Y.-S. Lee, and M. C. Downer, “Timeresolved electron-temperature measurement in a highly excited gold target using femtosecond thermionic emission," Physical Review B, vol. 50, no. 11, pp. 8016-8019, 1994. 
[19] D. A. B. Miller, Quantum Mechanics for Scientists and Engineers, Cambridge University Press, Cambridge, UK, 2008.

[20] R. H. Fowler and L. Nordheim, "Electron emission in intense electric fields," Proceedings of the Royal Society A, vol. 119, pp. 173-181, 1928.

[21] M. Cardona and L. Ley, Eds., Photoemission in Solids, Springer, Berlin, Germany, 1978.

[22] P. Hommelhoff, Y. Sortais, A. Aghajani-Talesh, and M. A. Kasevich, "Field emission tip as a nanometer source of free electron femtosecond pulses," Physical Review Letters, vol. 96, no. 7, Article ID 077401, 4 pages, 2006.

[23] L. V. Keldysh, "Ionization in the field of a strong electromagnetic wave," Journal of Experimental and Theoretical Physics, vol. 20, p. 1037, 1965.

[24] T. Brabec and F. Krausz, "Intense few-cycle laser fields: frontiers of nonlinear optics," Reviews of Modern Physics, vol. 72, pp. 545591,2000

[25] V. S. Popov, "Tunnel and multiphoton ionization of atoms and ions in a strong laser field (Keldysh theory)," Physics-Uspekhi, vol. 47, no. 9, pp. 855-885, 2004.

[26] P. Hommelhoff, C. Kealhofer, A. Aghajani-Talesh, Y. R. P. Sortais, S. M. Foreman, and M. A. Kasevich, "Extreme localization of electrons in space and time," Ultramicroscopy, vol. 109, no. 5, pp. 423-429, 2009.

[27] T. Topcu and F. Robicheaux, "Dichotomy between tunneling and multiphoton ionization in atomic photoionization: Keldysh parameter $\gamma$ versus scaled frequency $\Omega$," Physical Review A, vol. 86, no. 5, Article ID 053407, 10 pages, 2012.

[28] H. S. Kim, M. L. Yu, M. G. R. Thomson, E. Kratschmer, and T. H. P. Chang, "Energy distributions of $\mathrm{Zr} / \mathrm{O} / \mathrm{W}$ Schottky electron emission," Journal of Applied Physics, vol. 81, no. 1, pp. 461-465, 1997.

[29] Y. Sun, D. A. Jaffray, and J. T. W. Yeow, "Self-heating Schottky emission from a ballasted Carbon nanotube array," Carbon, vol. 58, pp. 87-91, 2013.

[30] K. L. Jensen, D. W. Feldman, and P. G. O'Shea, “Time dependent models of field-assisted photoemission," Journal of Vacuum Science \& Technology B, vol. 23, no. 2, pp. 621-631, 2005.

[31] J. W. Schwede, I. Bargatin, D. C. Riley et al., "Photon-enhanced thermionic emission for solar concentrator systems," Nature Materials, vol. 9, pp. 762-767, 2010.

[32] A. Nojeh, W.-K. Wong, A. W. Baum, R. F. Pease, and H. Dai, "Scanning electron microscopy of field-emitting individual single-walled Carbon nanotubes," Applied Physics Letters, vol. 85, no. 1, pp. 112-114, 2004.

[33] A. Nojeh, W.-K. Wong, E. Yieh, R. Fabian Pease, and H. Dai, "Electron beam stimulated field-emission from single-walled Carbon nanotubes," Journal of Vacuum Science \& Technology B, vol. 22, no. 6, pp. 3124-3127, 2004.

[34] C. W. Oatley and T. E. Everhart, "The examination of $\mathrm{p}-\mathrm{n}$ junctions with the scanning electron microscope," Journal of Electronics and Control, vol. 2, no. 6, pp. 568-570, 1957.

[35] K. Nakamae, H. Fujioka, and K. Ura, "Local field effects on voltage contrast in the scanning electron microscope," Journal of Physics D, vol. 14, no. 11, pp. 1939-1960, 1981.

[36] C. W. Oatley, "The early history of the scanning electron microscope," Journal of Applied Physics, vol. 53, no. 2, pp. R1R13, 1982.

[37] O. W. Richardson, "Electron emission from metals as a function of temperature," Physical Review, vol. 23, no. 2, pp. 153-155, 1924.
[38] S. Dushman, "Electron emission from metals as a function of temperature," Physical Review, vol. 21, no. 6, pp. 623-636, 1923.

[39] R. E. Burgess, H. Kroemer, and J. M. Houston, "Corrected values of Fowler-Nordheim field emission functions v(y) and $\mathrm{s}(\mathrm{y})$," Physical Review, vol. 90, no. 4, p. 515, 1953.

[40] E. L. Murphy and R. H. Good Jr., "Thermionic emission, field emission, and the transition region," Physical Review, vol. 102, no. 6, pp. 1464-1473, 1956.

[41] R. G. Forbes, "Refining the application of Fowler-Nordheim theory," Ultramicroscopy, vol. 79, no. 1-4, pp. 11-23, 1999.

[42] R. G. Forbes, "Field emission: New theory for the derivation of emission area from a Fowler-Nordheim plot," Journal of Vacuum Science \& Technology B, vol. 17, no. 2, pp. 526-533, 1999.

[43] R. G. Forbes, "Use of a spreadsheet for Fowler-Nordheim equation calculations," Journal of Vacuum Science \& Technology $B$, vol. 17, no. 2, pp. 534-541, 1999.

[44] R. G. Forbes and K. L. Jensen, "New results in the theory of Fowler-Nordheim plots and the modelling of hemi-ellipsoidal emitters," Ultramicroscopy, vol. 89, no. 1-3, pp. 17-22, 2001.

[45] R. G. Forbes, "Use of energy-space diagrams in free-electron models of field electron emission," Surface and Interface Analysis, vol. 36, pp. 395-401, 2004.

[46] R. G. Forbes, "Simple good approximations for the special elliptic functions in standard Fowler-Nordheim tunneling theory for a Schottky-Nordheim barrier," Applied Physics Letters, vol. 89, Article ID 113122, 2006.

[47] R. G. Forbes and J. H. B. Deane, "Reformulation of the standard theory of Fowler-Nordheim tunnelling and cold field electron emission," Proceedings of the Royal Society A, vol. 463, pp. 29072927, 2007.

[48] R. G. Forbes and J. H. B. Deane, "Comparison of approximations for the principal Schottky-Nordheim barrier function $\mathrm{v}(\mathrm{f})$, and comments on Fowler-Nordheim plots," Journal of Vacuum Science \& Technology B, vol. 28, no. 2, Article ID C2A33, 2010.

[49] R. G. Forbes, "Thin-slab model for field electron emission," Journal of Vacuum Science \& Technology B, vol. 28, no. 2, Article ID C2A43, 2010.

[50] R. G. Forbes, A. Fischer, and M. S. Mousa, "Improved approach to Fowler-Nordheim plot analysis," Journal of Vacuum Science \& Technology B, vol. 31, Article ID 02B103, 2013.

[51] A. Fischer, M. S. Mousa, and R. G. Forbes, "Influence of barrier form on Fowler-Nordheim plot analysis," Journal of Vacuum Science \& Technology B, vol. 31, Article ID 032201, 2013.

[52] R. H. Fowler, "The analysis of photoelectric sensitivity curves for clean metals at various temperatures," Physical Review, vol. 38, no. 1, pp. 45-56, 1931.

[53] L. A. DuBridge, "Theory of the energy distribution of photoelectrons," Physical Review, vol. 43, no. 9, pp. 727-741, 1933.

[54] J. H. Bechtel, W. Lee Smith, and N. Bloembergen, "Twophoton photoemission from metals induced by picosecond laser pulses," Physical Review B, vol. 15, no. 10, pp. 4557-4563, 1977.

[55] W. E. Spicer, "Photoemissive, photoconductive, and optical absorption studies of alkali-antimony compounds," Physical Review, vol. 112, no. 1, pp. 114-122, 1958.

[56] W. E. Spicer and A. Herrera-Gomez, "Modern theory and applications of photocathodes," in International Symposium on Optics, Imaging and Instrumentation, Proceedings of SPIE, San Diego, Calif, USA, July 1993. 
[57] K. L. Jensen, "General formulation of thermal, field, and photoinduced electron emission," Journal of Applied Physics, vol. 102, Article ID 024911, 2007.

[58] R. Browning, T. Z. Li, B. Chui et al., "Empirical forms for the electron/atom elastic scattering cross sections from 0.1 to 30 keV," Journal of Applied Physics, vol. 76, no. 4, pp. 2016-2022, 1994.

[59] D. C. Joy and S. Luo, "An empirical stopping power relationship for low-energy electrons," Scanning, vol. 11, pp. 176-180, 1989.

[60] P. J. F. Harris, Carbon Nanotube Science: Synthesis, Properties and Applications, Cambridge University Press, Cambridge, UK, 2009.

[61] A. Javey and J. Kong, Eds., Carbon Nanotube Electronics, Springer, New York, NY, USA, 2009.

[62] S. Chopra, K. McGuire, N. Gothard, A. M. Rao, and A. Pham, "Selective gas detection using a Carbon nanotube sensor," Applied Physics Letters, vol. 83, no. 11, pp. 2280-2282, 2003.

[63] R. A. MacDonald, B. F. Laurenzi, G. Viswanathan, P. M. Ajayan, and J. P. Stegemann, "Collagen-Carbon nanotube composite materials as scaffolds in tissue engineering," Journal of Biomedical Materials Research A, vol. 74, no. 3, pp. 489-496, 2005.

[64] L. Yang, L. Yang, S. Wang, Q. Zeng, Z. Zhang, and L. M. Peng, "Carbon nanotube photoelectronic and photovoltaic devices and their applications in infrared detection," Small, vol. 9, no. 8, pp. 1225-1236, 2013.

[65] A. J. Miller, R. A. Hatton, and S. R. P. Silva, "Watersoluble multiwall-Carbon-nanotube-polythiophene composite for bilayer photovoltaics," Applied Physics Letters, vol. 89, Article ID 123115, 2006.

[66] S. Sotiropoulou and N. A. Chaniotakis, "Carbon nanotube array-based biosensor," Analytical and Bioanalytical Chemistry, vol. 375, no. 1, pp. 103-105, 2003.

[67] Y. Saito, Ed., Carbon Nanotube and Related Field EmittersFundamentals and Applications, Wiley-VCH, Weinheim, Germany, 2010

[68] B. Zhao, D. N. Futaba, S. Yasuda, M. Akoshima, T. Yamada, and K. Hata, "Exploring advantages of diverse Carbon nanotube forests with tailored structures synthesized by supergrowth from engineered catalysts," ACS Nano, vol. 3, no. 1, pp. 108-114, 2009.

[69] K. Hasegawa and S. Noda, "Millimeter-tall single-walled Carbon nanotubes rapidly grown with and without water," ACS Nano, vol. 5, no. 2, pp. 975-984, 2011.

[70] P. L. McEuen, M. S. Fuhrer, and H. Park, "Single-walled Carbon nanotube electronics," IEEE Transactions on Nanotechnology, vol. 1, no. 1, pp. 78-84, 2002.

[71] N. de Jonge and J.-M. Bonard, "Carbon nanotube electron sources and applications," Philosophical Transactions of the Royal Society A, vol. 362, p. 2239, 2004.

[72] A. G. Rinzler, J. H. Hafner, P. Nikolaev et al., "Unraveling nanotubes: field emission from an atomic wire," Science, vol. 269, no. 5230, pp. 1550-1553, 1995.

[73] W. A. de Heer, A. Châtelain, and D. Ugarte, "A Carbon nanotube field-emission electron source," Science, vol. 270, no. 5239, pp. 1179-1180, 1995.

[74] P. G. Collins and A. Zettl, "A simple and robust electron beam source from Carbon nanotubes," Applied Physics Letters, vol. 69, no. 13, pp. 1969-1971, 1996.

[75] J.-M. Bonard, J.-P. Salvetat, T. Stöckli, W. A. De Heer, L. Forró, and A. Chatelain, "Field emission from single-wall Carbon nanotube films," Applied Physics Letters, vol. 73, no. 7, pp. 918920, 1998.
[76] K. A. Dean and B. R. Chalamala, "Current saturation mechanisms in Carbon nanotube field emitters," Applied Physics Letters, vol. 76, no. 3, pp. 375-377, 2000.

[77] A. Nojeh and R. F. W. Pease, "Field-electron emission from single-walled Carbon nanotubes lying on a surface," in Proceedings of the Canadian Conference on Electrical and Computer Engineering, pp. 1294-1297, Vancouver, Canada, April 2007.

[78] R. K. Joshi, J. Engstler, A. Navitski, V. Sakharuk, G. Müller, and J. J. Schneider, "Gas phase synthesis and field emission properties of 3D aligned double walled Carbon nanotube/anatase hybrid architectures," Nanoscale, vol. 3, no. 8, pp. 3258-3264, 2011.

[79] S. Fan, M. G. Chapline, N. R. Franklin, T. W. Tombler, A. M. Cassell, and H. Dai, "Self-oriented regular arrays of Carbon nanotubes and their field emission properties," Science, vol. 283, no. 5401, pp. 512-514, 1999.

[80] D. S. Y. Hsu and J. L. Shaw, "Integrally gated Carbon nanotubeon-post field emitter arrays," Applied Physics Letters, vol. 80, no. 1, pp. 118-120, 2002.

[81] D. S. Y. Hsu and J. L. Shaw, "Robust and regenerable integrally gated Carbon nanotube field emitter arrays," Journal of Applied Physics, vol. 98, Article ID 014314, 2005.

[82] H. M. Manohara, M. J. Bronikowski, M. Hoenk, B. D. Hunt, and P. H. Siegel, "High-current-density field emitters based on arrays of Carbon nanotube bundles," Journal of Vacuum Science \& Technology B, vol. 23, no. 1, pp. 157-161, 2005.

[83] D. Lysenkov, H. Abbas, G. Müller, J. Engstler, K. P. Budna, and J. J. Schneider, "Electron field emission from Carbon nanotubes on porous alumina," Journal of Vacuum Science \& Technology B, vol. 23, no. 2, pp. 809-813, 2005.

[84] W. I. Milne, K. B. K. Teo, G. A. J. Amaratung et al., "Carbon nanotubes as field emission sources," Journal of Materials Chemistry, vol. 14, pp. 933-943, 2004.

[85] W. I. Milne, K. B. K. Teo, M. Mann et al., "Carbon nanotubes as electron sources," Physica Status Solidi (a), vol. 203, pp. 10581063, 2006.

[86] N. Sinha, D. Roy Mahapatra, Y. Sun, J. T. Yeow, R. V. Melnik, and D. A. Jaffray, "Electromechanical interactions in a Carbon nanotube based thin film field emitting diode," Nanotechnology, vol. 19, no. 2, Article ID 025701, 2008.

[87] Y. Saito, K. Hamaguchi, T. Nishino et al., "Field emission patterns from single-walled Carbon nanotubes," Japanese Journal of Applied Physics 2, vol. 36, no. 10, pp. L1340-L1342, 1997.

[88] Y. Saito, T. Matsukawa, K. Asaka, and H. Nakahara, "Field emission microscopy of Al-deposited Carbon nanotubes: emission stability improvement and image of an $\mathrm{Al}$ atom cluster," Journal of Vacuum Science \& Technology B, vol. 28, no. 2, Article ID C2A5, 2010.

[89] Q. H. Wang, A. A. Setlur, J. M. Lauerhaas, J. Y. Dai, E. W. Seelig, and R. P. H. Chang, "A nanotube-based field-emission flat panel display," Applied Physics Letters, vol. 72, no. 22, pp. 2912-2913, 1998.

[90] W. B. Choi, D. S. Chung, J. H. Kang et al., "Fully sealed, highbrightness Carbon-nanotube field-emission display," Applied Physics Letters, vol. 75, no. 20, pp. 3129-3131, 1999.

[91] J. L. Kwo, M. Yokoyama, W. C. Wang, F. Y. Chuang, and I. N. Lin, "Characteristics of flat panel display using Carbon nanotubes as electron emitters," Diamond and Related Materials, vol. 9, pp. 1270-1274, 2000.

[92] S. G. Yu, S. H. Jin, W. K. Yi et al., "Undergate-type triode Carbon nanotube field emission display with a microchannel plate," Japanese Journal of Applied Physics, vol. 40, pp. 6088-6091, 2001. 
[93] N. de Jonge, "Carbon nanotube electron sources for electron microscopes," Advances in Imaging \& Electron Physics, vol. 156, pp. 2159-2164, 2009.

[94] R. Yabushita, K. Hata, H. Sato, and Y. Saito, "Development of compact field emission scanning electron microscope equipped with multiwalled Carbon nanotube bundle cathode," Journal of Vacuum Science \& Technology B, vol. 25, no. 2, pp. 640-642, 2007.

[95] M. Mann, M. El Gomati, T. Wells, W. I. Milne, and K. B. K. Teo, "The application of Carbon nanotube electron sources to the electron microscope," in Carbon Nanotubes and Associated Devices, vol. 7037 of Proceedings of SPIE, p. 6, 2008.

[96] S. A. Getty, T. T. King, R. A. Bis et al., "Performance of a Carbon nanotube field emission electron gun," in Micro (MEMS) and Nanotechnologies for Defense and Security, vol. 6556 of Proceedings of SPIE, 2007.

[97] M. Croci, I. Arfaoui, T. Stöckli, A. Chátelain, and J.-M. Bonard, "A fully sealed luminescent tube based on Carbon nanotube field emission," Microelectronics Journal, vol. 35, no. 4, pp. 329336, 2004.

[98] Y. Cheng, J. Zhang, Y. Z. Lee et al., "Dynamic radiography using a Carbon-nanotube-based field-emission x-ray source," Review of Scientific Instruments, vol. 75, p. 3264, 2004.

[99] N. Sinha and J. T.-W. Yeow, "Carbon nanotubes for biomedical applications," IEEE Transactions on Nanobioscience, vol. 4, no. 2, pp. 180-195, 2005.

[100] K. Kawakita, K. Hata, H. Sato, and Y. Saito, "Development of microfocused $\mathrm{x}$-ray source by using Carbon nanotube field emitter," Journal of Vacuum Science \& Technology B, vol. 24, no. 2, pp. 950-952, 2006.

[101] P. von Allmen, L. R. C. Fonseca, and R. Ramprasad, "Calculating the field emission current from a Carbon nanotube," Physica Status Solidi (b), vol. 226, no. 1, pp. 107-113, 2001.

[102] H. Shimoyama, H. Murata, and T. Ohye, "Computer simulation of electric field anaysis for vertically aligned Carbon nanotubes (2) - electric field on the nanotube apex," in Charged Particle Detection, Diagnostics, and Imaging, vol. 4510 of Proceedings of SPIE, pp. 163-171, August 2001.

[103] S. Han, Theoretical Study on the Field Emission of Carbon Nanotubes, Seoul National University, 2000.

[104] G. Zhou and Y. Kawazoe, "Localized valence states characteristics and work function of single-walled Carbon nanotubes: a first-principles study," Physical Review B, vol. 65, no. 15, Article ID 155422, 5 pages, 2002.

[105] G. Zhou, W. Duan, and B. Gu, "Electronic structure and fieldemission characteristics of open-ended single-walled Carbon nanotubes," Physical Review Letters, vol. 87, no. 9, Article ID 95504, 4 pages, 2001.

[106] C. Kim, B. Kim, S. M. Lee, C. Jo, and Y. H. Lee, "Electronic structures of capped Carbon nanotubes under electric fields," Physical Review B, vol. 65, Article ID 165418, 2002.

[107] J. Luo, L.-M. Peng, Z. Q. Xue, and J. L. Wu, "End potential barriers of single-walled Carbon nanotubes and their role in field emission," Physical Review B, vol. 66, Article ID 155407, 5 pages, 2002.

[108] X. Zheng, G. H. Chen, Z. Li, S. Deng, and N. Xu, "Quantummechanical investigation of field-emission mechanism of a micrometer-long single-walled Carbon nanotube," Physical Review Letters, vol. 92, no. 10, Article ID 106803, 4 pages, 2004.
[109] P. Yaghoobi, K. Walus, and A. Nojeh, "First-principles study of quantum tunneling from nanostructures: current in a singlewalled Carbon nanotube electron source," Physical Review B, vol. 80, no. 11, Article ID 115422, 7 pages, 2009.

[110] P. Yaghoobi, M. K. Alam, K. Walus, and A. Nojeh, "High subthreshold field-emission current due to Hydrogen adsorption in single-walled Carbon nanotubes: a first-principles study," Applied Physics Letters, vol. 95, no. 26, Article ID 262102, 2009.

[111] A. Kashefian Naieni, P. Yaghoobi, and A. Nojeh, "Firstprinciples study of field-emission from Carbon nanotubes in the presence of methane," Journal of Vacuum Science \& Technology B, vol. 30, Article ID 021803, 7 pages, 2012.

[112] J.-M. Bonard, H. Kind, T. Stickli, and L.-O. Nilsson, "Field emission from Carbon nanotubes: the first five years," SolidState Electronics, vol. 45, pp. 893-914, 2001.

[113] P. Yaghoobi and A. Nojeh, "Electron emission from Carbon nanotubes," Modern Physics Letters B, vol. 21, no. 27, pp. 18071830, 2007.

[114] Y. Saito, K. Hamaguchi, R. Mizushima et al., "Field emission from Carbon nanotubes and its application to cathode ray tube lighting elements," Applied Surface Science, vol. 146, pp. 305-311, 1999.

[115] Y. Saito and S. Uemura, "Field emission from Carbon nanotubes and its application to electron sources," Carbon, vol. 38, no. 2, pp. 169-182, 2000.

[116] P. G. Collins and A. Zettl, "Unique characteristics of cold cathode Carbon-nanotube-matrix field emitters," Physical Review B, vol. 55, no. 15, pp. 9391-9399, 1997.

[117] S.-D. Liang and L. Chen, "Theories of field and thermionic electron emissions from Carbon nanotubes," Journal of Vacuum Science \& Technology B, vol. 28, no. 2, Article ID C2A50, 2010.

[118] J. Peng, Z. Li, C. He et al., "The roles of apex dipoles and field penetration in the physics of charged, field emitting, singlewalled Carbon nanotubes," Journal of Applied Physics, vol. 104, Article ID 014310, 2008.

[119] P. A. Zestanakis and J. P. Xanthakis, "Field emission from open multiwall Carbon nanotubes: a case of non-Fowler-Nordheim behavior," Journal of Applied Physics, vol. 104, no. 9, Article ID 094312, 2008.

[120] J. P. Xanthakis, G. C. Kokkorakis, and E. I. Sfakianakis, "Angular variation of the transmission coefficient of electrons from open Carbon nanotubes by a 3-dimensional WKB method," Physica E, vol. 40, no. 4, pp. 873-877, 2008.

[121] A. Buldum and J. P. Lu, "Electron field Emission properties of closed Carbon nanotubes," Physical Review Letters, vol. 91, no. 23, Article ID 236801, 4 pages, 2003.

[122] D. C. Joy, Monte Carlo Modeling for Electron Microscopy and Microanalysis, Oxford University Press, New York, NY, USA, 1995.

[123] P. Finnie, K. Kaminska, Y. Homma, D. G. Austing, and J. Lefebvre, "Charge contrast imaging of suspended nanotubes by scanning electron microscopy," Nanotechnology, vol. 19, no. 33, Article ID 335202, 2008.

[124] T. Brintlinger, Y.-F. Chen, T. Dürkop et al., "Rapid imaging of nanotubes on insulating substrates," Applied Physics Letters, vol. 81, no. 13, pp. 2454-2456, 2002.

[125] W. K. Wong, A. Nojeh, and R. F. W. Pease, "Parameters and mechanisms governing image contrast in scanning electron microscopy of single-walled Carbon nanotubes," Scanning, vol. 28, no. 4, pp. 219-227, 2006. 
[126] Y. Homma, S. Suzuki, Y. Kobayashi, M. Nagase, and D. Takagi, "Mechanism of bright selective imaging of single-walled Carbon nanotubes on insulators by scanning electron microscopy," Applied Physics Letters, vol. 84, no. 10, pp. 1750-1752, 2004.

[127] Y. A. Kasumov, I. I. Khodos, M. Kociak, and A. Y. Kasumov, "Scanning and transmission electron microscope images of a suspended single-walled Carbon nanotube," Applied Physics Letters, vol. 89, no. 1, Article ID 013120, 2006.

[128] M. K. Alam, S. P. Eslami, and A. Nojeh, "Secondary electron emission from single-walled Carbon nanotubes," Physica E, vol. 42, no. 2, pp. 124-131, 2009.

[129] J. Luo, J. H. Warner, C. Feng et al., "Ultrahigh secondary electron emission of Carbon nanotubes," Applied Physics Letters, vol. 96, Article ID 213113, 2010.

[130] M. K. Alam, R. F. W. Pease, and A. Nojeh, "Comment on "ultrahigh secondary electron emission of Carbon nanotubes" [Appl. Phys. Lett., vol. 96, 213113, 2010]," Applied Physics Letters, vol. 98, Article ID 066101, 2011.

[131] A. Nojeh, B. Shan, K. Cho, and R. F. W. Pease, "Ab Initio modeling of the interaction of electron beams and single-walled Carbon nanotubes," Physical Review Letters, vol. 96, no. 5, Article ID 056802, 4 pages, 2006.

[132] M. K. Alam and A. Nojeh, "Monte Carlo simulation of electron scattering and secondary electron emission in individual multiwalled Carbon nanotubes: a discrete-energy-loss approach," Journal of Vacuum Science \& Technology B, vol. 29, no. 4, Article ID 041803, 2011.

[133] M. K. Alam, P. Yaghoobi, M. Chang, and A. Nojeh, "Secondary electron yield of multiwalled Carbon nanotubes," Applied Physics Letters, vol. 97, Article ID 261902, 2010.

[134] I. Kyriakou, D. Emfietzoglou, R. Garcia-Molina, I. Abril, and K. Kostarelos, "Electron inelastic mean free paths for Carbon nanotubes from optical data," Applied Physics Letters, vol. 94, Article ID 263113, 2009.

[135] I. Kyriakou, D. Emfietzoglou, R. Garcia-Molina, I. Abril, and K. Kostarelos, "Simple model of bulk and surface excitation effects to inelastic scattering in low-energy electron beam irradiation of multi-walled Carbon nanotubes," Journal of Applied Physics, vol. 110, Article ID 054304, 2011.

[136] D. Emfietzoglou, I. Kyriakou, R. Garcia-Molina, I. Abril, and K. Kostarelos, "Quasi first-principles Monte Carlo modeling of energy dissipation by low-energy electron beams in multiwalled Carbon nanotube materials," Applied Physics Letters, vol. 100, no. 9, Article ID 093113, 2012.

[137] D. Emfietzoglou, I. Kyriakou, R. Garcia-Molina, I. Abril, and K. Kostarelos, "Analytic expressions for the inelastic scattering and energy loss of electron and proton beams in Carbon nanotubes," Journal of Applied Physics, vol. 108, Article ID 054312, 2010.

[138] M. K. Alam, P. Yaghoobi, and A. Nojeh, "Unusual secondary electron emission behavior in Carbon nanotube forests," Scanning, vol. 31, no. 6, pp. 221-228, 2009.

[139] M. K. Alam, P. Yaghoobi, and A. Nojeh, "Monte Carlo modeling of electron backscattering from Carbon nanotube forests," Journal of Vacuum Science \& Technology B, vol. 28, no. 6, Article ID C6J13, 2010.

[140] I. Kyriakou, D. Emfietzoglou, A. Nojeh, and M. Moscovitch, "Monte Carlo study of electron beam penetration and backscattering in multi-walled Carbon nanotube materials: the effect of different scattering models," Journal of Applied Physics, vol. 113, Article ID 084303, 2013.

[141] D. Emfietzoglou, G. Papamichael, and M. Moscovitch, "Eventby-event computer simulation of interactions of energetic charged particles and all their secondary electrons in water," Journal of Physics D, vol. 33, no. 8, pp. 932-944, 2000.

[142] M. Michan, P. Yaghoobi, B. Wong, and A. Nojeh, "High electron gain from single-walled Carbon nanotubes stimulated by interaction with an electron beam," Physical Review B, vol. 81, no. 19, Article ID 195438, 8 pages, 2010.

[143] M. Michan and A. Nojeh, "High electron gain from forests of multi-walled Carbon nanotubes," Materials Research Society Symposium Proceedings, vol. 1258, pp. 295-302, 2010.

[144] A. V. Krasheninnikov, Y. Miyamoto, and D. Tománek, "Role of electronic excitations in ion collisions with Carbon nanostructures," Physical Review Letters, vol. 99, no. 1, Article ID 016104, 4 pages, 2007.

[145] I. Kyriakou, C. Celedon, R. Segura et al., "Energy loss of protons in Carbon nanotubes: experiments and calculations," Nuclear Instruments and Methods in Physics Research B, vol. 268, no. 1112, pp. 1781-1785, 2010.

[146] J. E. Valdés, C. Celedan, R. Segura et al., "Energy loss distribution of proton beams at normal incidence on multi-walled Carbon nanotubes," Carbon, vol. 52, pp. 137-144, 2013.

[147] A. V. Krasheninnikov and F. Banhart, "Engineering of nanostructured Carbon materials with electron or ion beams," Nature Materials, vol. 6, no. 10, pp. 723-733, 2007.

[148] X. Wei, D. Golberg, Q. Chen, Y. Bando, and L. Peng, "Phononassisted electron emission from individual Carbon nanotubes," Nano Letters, vol. 11, no. 2, pp. 734-739, 2011.

[149] P. Liu, Y. Wei, K. Jiang et al., "Thermionic emission and work function of multiwalled Carbon nanotube yarns," Physical Review B, vol. 73, no. 23, Article ID 235412, 5 pages, 2006.

[150] Z. Xu, X. D. Bai, E. G. Wang, and Z. L. Wang, "Field emission of individual Carbon nanotubes in the scanning electron microscope," Applied Physics Letters, vol. 87, Article ID 163106, 2005.

[151] L. Fleming, M. D. Ulrich, K. Efimenko et al., "Near-edge absorption fine structure and UV photoemission spectroscopy studies of aligned single-walled Carbon nanotubes on $\mathrm{Si}(100)$ substrates," Journal of Vacuum Science \& Technology B, vol. 22, p. $2000,2004$.

[152] H. Shiozawa, H. Ishii, H. Kataura et al., "Photoemission spectroscopy on single-wall Carbon nanotubes," Physica B, vol. 351, no. 3, pp. 259-261, 2004.

[153] R. Larciprete, A. Goldonia, S. Lizzita, and L. Petacciaa, “The electronic properties of Carbon nanotubes studied by high resolution photoemission spectroscopy," Applied Surface Science, vol. 248, pp. 8-13, 2005.

[154] C. Bittencourt, A. Feltena, B. Douhard et al., "Photoemission studies of gold clusters thermally evaporated on multiwall Carbon nanotubes," Chemical Physics, vol. 328, pp. 385-391, 2006.

[155] B. Ha, J. Park, S. Y. Kim, and C. J. Lee, "Investigation of field emission and photoemission properties of high-purity single-walled Carbon nanotubes synthesized by Hydrogen arc discharge," The Journal of Physical Chemistry B, vol. 110, no. 47, pp. 23742-23749, 2006.

[156] N. Kocharova, J. Leiro, J. Lukkari et al., "Self-assembled Carbon nanotubes on gold: Polarization-modulated infrared reflectionabsorption spectroscopy, high-resolution X-ray photoemission spectroscopy, and near-edge X-ray absorption fine structure spectroscopy study," Langmuir, vol. 24, no. 7, pp. 3235-3243, 2008. 
[157] S. Suzuki, Y. Watanabe, T. Ogino et al., "Observation of singlewalled Carbon nanotubes by photoemission microscopy," Carbon, vol. 42, no. 3, pp. 559-563, 2004.

[158] S. Suzuki, Y. Watanabe, Y. Homma, S. Y. Fukuba, A. Locatelli, and S. Heun, "Photoemission electron microscopy of individual single-walled Carbon nanotubes," Journal of Electron Spectroscopy and Related Phenomena, vol. 144-147, pp. 357-360, 2005.

[159] T. H. Wong, M. C. Gupta, and C. Hernandez-Garcia, "Nanosecond laser pulse-induced electron emission from multiwall Carbon nanotube film," Nanotechnology, vol. 18, Article ID 135705, 2007.

[160] P. Yaghoobi, M. Michan, and A. Nojeh, "Middle-ultraviolet laser photoelectron emission from vertically aligned millimeter-long multiwalled Carbon nanotubes," Applied Physics Letters, vol. 97, Article ID 153119, 2010.

[161] A. Nojeh, K. Ioakeimidi, S. Sheikhaei, and R. F. W. Pease, "Photoemission from single-walled Carbon nanotubes," Journal of Applied Physics, vol. 104, no. 5, Article ID 054308, 2008.

[162] K. Kempa, J. Rybczynski, Z. Huang et al., "Carbon nanotubes as optical antennae," Advanced Materials, vol. 19, no. 3, pp. 421426, 2007.

[163] G. W. Hanson, "Fundamental transmitting properties of Carbon nanotube antennas," IEEE Transactions on Antennas and Propagation, vol. 53, no. 11, pp. 3426-3435, 2005.

[164] P. J. Burke, S. Li, and Z. Yu, "Quantitative theory of nanowire and nanotube antenna performance," IEEE Transactions on Nanotechnology, vol. 5, no. 4, pp. 314-334, 2006.

[165] G. Y. Slepyan, M. V. Shuba, S. A. Maksimenko, and A. Lakhtakia, "Theory of optical scattering by achiral Carbon nanotubes and their potential as optical nanoantennas," Physical Review B, vol. 73, no. 19, Article ID 195416, 11 pages, 2006.

[166] Y. Wang, K. Kempa, B. Kimball et al., "Receiving and transmitting light-like radio waves: antenna effect in arrays of aligned Carbon nanotubes," Applied Physics Letters, vol. 85, no. 13, pp. 2607-2609, 2004.

[167] A. Nojeh, P. Pande, A. Ganguly, S. Sheikhaei, B. Belzer, and A. Ivanov, "Reliability of wireless on-chip interconnects based on Carbon nanotube antennas," in Proceedings of the 14th IEEE International Mixed-Signals, Sensors and Systems Test Workshop, pp. 1-6, Vancouver, Canada, 2008.

[168] A. Nojeh and A. Ivanov, "Wireless interconnect and the potential for Carbon nanotubes," IEEE Design and Test of Computers, vol. 27, no. 4, pp. 44-52, 2010.

[169] J. R. Maldonado, S. T. Coyle, J. K. Varner, R. C. Moore, P. R. H. Stark, and D. N. Larson, "Preliminary evaluation of surface plasmon enhanced light transmission with a scanning 257 nm ultraviolet microscope," Journal of Vacuum Science \& Technology B, vol. 22, no. 6, pp. 3552-3556, 2004.

[170] H. J. Choi and T. R. Groves, "Analysis of surface electromagnetic wave resonant structures for potential application in an array of compact photoelectron sources," Journal of Vacuum Science \& Technology B, vol. 28, no. 6, Article ID C6C63, 2010.

[171] R. K. Li, H. To, G. Andonian et al., "Surface-plasmon resonanceenhanced multiphoton emission of high-brightness electron beams from a nanostructured Copper cathode," Physical Review Letters, vol. 110, no. 7, Article ID 074801, 5 pages, 2013.

[172] A. Polyakov, C. Senft, K. F. Thompson et al., "Plasmonenhanced photocathode for high brightness and high repetition rate X-Ray sources," Physical Review Letters, vol. 110, no. 7, Article ID 076802, 5 pages, 2013.
[173] P. Dombi, A. Hörl, P. Rácz et al., "Ultrafast strong-field photoemission from plasmonic nanoparticles," Nano Letters, vol. 13, no. 2, pp. 674-678, 2013.

[174] P. M. Nagel, J. S. Robinsonc, B. D. Harteneck et al., "Surface plasmon assisted electron acceleration in photoemission from gold nanopillars," Chemical Physics, vol. 414, pp. 106-111, 2013.

[175] P. Yaghoobi, M. V. Moghaddam, M. Michan, and A. Nojeh, "Visible-light induced electron emission from Carbon nanotube forests," Journal of Vacuum Science \& Technology B, vol. 29, no. 2, Article ID 02B104, 2011.

[176] D. C. Cox, R. D. Forrest, P. R. Smith, and S. R. P. Silva, "Thermionic emission from defective Carbon nanotubes," Applied Physics Letters, vol. 85, no. 11, pp. 2065-2067, 2004.

[177] P. Liu, Q. Sun, F. Zhu et al., "Measuring the work function of Carbon nanotubes with thermionic method," Nano Letters, vol. 8, pp. 647-651, 2008.

[178] P. Liu, Y. Wei, K. Jiang et al., "Thermionic emission and work function of multiwalled Carbon nanotube yarns," Physical Review B, vol. 73, no. 23, Article ID 235412, 5 pages, 2006.

[179] Y. Wei, K. Jiang, X. Feng et al., "Comparative studies of multiwalled Carbon nanotube sheets before and after shrinking," Physical Review B, vol. 76, Article ID 045423, 7 pages, 2007.

[180] S. T. Purcell, P. Vincent, C. Journet, and V. Thien Binh, "Hot nanotubes: stable heating of individual multiwall Carbon nanotubes to $2000 \mathrm{~K}$ induced by the field-emission current," Physical Review Letters, vol. 88, no. 10, Article ID 105502, 4 pages, 2002.

[181] S. Berber, Y.-K. Kwon, and D. Tománek, "Unusually high thermal conductivity of Carbon nanotubes," Physical Review Letters, vol. 84, no. 20, pp. 4613-4616, 2000.

[182] J. Che, T. Çagin, and W. A. Goddard, "Thermal conductivity of Carbon nanotubes," Nanotechnology, vol. 11, no. 2, p. 65, 2000.

[183] P. Kim, L. Shi, A. Majumdar, and P. L. McEuen, "Thermal transport measurements of individual multiwalled nanotubes," Physical Review Letters, vol. 87, Article ID 215502, 4 pages, 2001.

[184] D. J. Yang, Q. Zhang, G. Chen et al., "Thermal conductivity of multiwalled Carbon nanotubes," Physical Review B, vol. 66, Article ID 165440, 2002.

[185] N. Mingo and D. A. Broido, "Carbon nanotube ballistic thermal conductance and its limits," Physical Review Letters, vol. 95, no. 9, Article ID 096105, 2005.

[186] Y. Gu and Y. Chen, "Thermal conductivities of single-walled Carbon nanotubes calculated from the complete phonon dispersion relations," Physical Review B, vol. 76, Article ID 134110, 9 pages, 2007.

[187] H. Huang, C. H. Liu, Y. Wu, and S. Fan, "Aligned Carbon nanotube composite films for thermal management," Advanced Materials, vol. 17, no. 13, pp. 1652-1656, 2005.

[188] P. Yaghoobi, M. V. Moghaddam, and A. Nojeh, “Heat trap' light-induced localized heating and thermionic electron emission from Carbon nanotube arrays," Solid State Communications, vol. 151, pp. 1105-1108, 2011.

[189] E. Pop, D. Mann, Q. Wang, K. Goodson, and H. Dai, “Thermal conductance of an individual single-wall Carbon nanotube above room temperature," Nano Letters, vol. 6, pp. 96-100, 2006.

[190] E. Pop, D. A. Mann, K. E. Goodson, and H. Dai, "Electrical and thermal transport in metallic single-wall Carbon nanotubes on insulating substrates," Journal of Applied Physics, vol. 101, Article ID 093710, 2007.

[191] E. Pop, D. Mann, J. Cao, Q. Wang, K. Goodson, and H. Dai, "Negative differential conductance and hot phonons in 
suspended nanotube molecular wires," Physical Review Letters, vol. 95, no. 15, Article ID 155505, 4 pages, 2005.

[192] M. A. Osman and D. Srivastava, "Temperature dependence of the thermal conductivity of single-wall Carbon nanotubes," Nanotechnology, vol. 12, no. 1, pp. 21-24, 2001.

[193] S. P. Hepplestone and G. P. Srivastava, "Phonon-phonon interactions in single-wall Carbon nanotubes," Physical Review B, vol. 74, Article ID 165420, 11 pages, 2006.

[194] P. Yaghoobi, M. Vahdani Moghaddam, and A. Nojeh, "Solar electron source and thermionic solar cell," AIP Advances, vol. 2, Article ID 042139, 2012.

[195] M. Chang, M. Vahdani Moghaddam, A. Khoshaman et al., "High temperature gradient in a conductor: Carbon nanotube forest under the "Heat Trap" condition," in Proceedings of the 57th International Conference on Electron, Ion, and Photon Beam Technology and Nanofabrication (EIPBN '13), Nashville, Tenn, USA, 2013.

[196] M. Vahdani Moghaddam, P. Yaghoobi, and A. Nojeh, "Polarization-dependent light-induced thermionic electron emission from Carbon nanotube arrays using a wide range of wavelengths," Applied Physics Letters, vol. 101, Article ID 253110, 2012.

[197] Z.-P. Yang, L. Ci, J. A. Bur, S. Y. Lin, and P. M. Ajayan, "Experimental observation of an extremely dark material made By a low-density nanotube array," Nano Letters, vol. 8, pp. 446451, 2008.

[198] K. Mizuno, J. Ishii, H. Kishida et al., "A black body absorber from vertically aligned single-walled Carbon nanotubes," Proceedings of the National Academy of Sciences of the United States of America, vol. 106, pp. 6044-6047, 2009.

[199] T. L. Westover, A. D. Franklin, B. A. Cola, T. S. Fisher, and R. G. Reifenberger, "Photo- and thermionic emission from potassium-intercalated Carbon nanotube arrays," Journal of Vacuum Science \& Technology B, vol. 28, no. 2, pp. 423-434, 2010.

[200] M. Vahdani Moghaddam, M. Sawar, Z. Xiao, M. Dahmardeh, K. Takahata, and A. Nojeh, "Non-linear photoemission from Carbon nanotube arrays," in Proceedings of the 26th International Vacuum Nanoelectronics Conference (IVNC '13), Roanoke, NC, USA, 2013.

[201] H. C. Pfeiffer, "New imaging and deflection concept for probeforming microfabrication systems," Journal of Vacuum Science \& Technology B, vol. 12, p. 1170, 1975.

[202] H. C. Pfeiffer, "Variable spot shaping for electron-beam lithography," Journal of Vacuum Science \& Technology B, vol. 15, p. 887, 1978.

[203] H. C. Pfeiffer, D. E. Davis, W. A. Enichen et al., "EL-4, a new generation electron-beam lithography system ," Journal of Vacuum Science \& Technology B, vol. 11, p. 2332, 1993.

[204] T. H. Newman, R. F. W. Pease, and W. DeVore, "Dot matrix electron beam lithography," Journal of Vacuum Science \& Technology B, vol. 1, no. 4, pp. 999-1002, 1983.

[205] H. Yasuda, S. Arai, J.-I. Kai et al., "Fast electron beam lithography system with 1024 beams individually controlled by blanking aperture array," Japanese Journal of Applied Physics 1, vol. 32, no. 12, pp. 6012-6017, 1993.

[206] T. H. P. Chang, M. G. R. Thomson, E. Kratschmer et al., "Electron-beam microcolumns for lithography and related applications," Journal of Vacuum Science \& Technology B, vol. 14, no. 6, pp. 3774-3781, 1996.
[207] J. E. Schneider, A. W. Baum, G. I. Winograd et al., "Semiconductor on glass photocathodes as high-performance sources for parallel electron beam lithography," Journal of Vacuum Science \& Technology B, vol. 14, no. 6, pp. 3782-3786, 1996.

[208] G. I. Winograd, R. F. W. Pease, and M. A. McCord, "Blanked aperture array for parallel electron beam lithography," Journal of Vacuum Science \& Technology B, vol. 15, no. 6, pp. 2289-2292, 1997.

[209] M. Mankos, S. Coyle, A. Fernandez et al., "Multisource optimization of a column for electron lithography," Journal of Vacuum Science \& Technology B, vol. 18, no. 6, pp. 3010-3016, 2000.

[210] D. S. Pickard, C. Campbell, T. Crane et al., "Distributed axis electron-beam system for lithography and inspectionpreliminary experimental results," Journal of Vacuum Science \& Technology B, vol. 20, no. 6, pp. 2662-2665, 2002.

[211] D. S. Pickard, T. R. Groves, W. D. Meisburger, T. Crane, and R. F. Pease, "Distributed axis electron beam technology for maskless lithography and defect inspection," Journal of Vacuum Science \& Technology B, vol. 21, no. 6, pp. 2834-2838, 2003.

[212] M. Vahdani Moghaddam and A. Nojeh, "Shaped and multiple electron beams from a single thermionic cathode," in Proceedings of the 57th International Conference on Electron, Ion, and Photon Beam Technology and Nanofabrication (EIPBN '13), Nashville, Tenn, USA, 2013.

[213] W. Schlichter, "Die spontane Elektronenemission glühender Metalle und das glühelektrische element," Annalen der Physik, vol. 352, pp. 573-640, 1915.

[214] V. C. Wilson, "Conversion of heat to electricity by thermionic emission," Journal of Applied Physics, vol. 30, no. 4, pp. 475-481, 1959.

[215] G. N. Hatsopoulos and E. P. Gyftopoulos, Thermionic Energy Conversion: Theory, Technology, and Application, MIT Press, 1979.

[216] A. Shakouri, "Thermoelectric, thermionic and thermophotovoltaic energy conversion," in Proceedings of the 24th International Conference on Thermoelectrics, pp. 507-512, 2005.

[217] Y. G. Deng and J. Liu, "Recent advances in direct solar thermal power generation," Journal of Renewable and Sustainable Energy, vol. 1, Article ID 052701, 2009.

[218] J.-H. Lee, I. Bargatin, N. A. Melosh, and R. T. Howe, "Optimal emitter-collector gap for thermionic energy converters," Applied Physics Letters, vol. 100, Article ID 173904, 2012.

[219] S. F. Adams, "Solar thermionic space power technology testing: a historical perspective," AIP Conference Proceedings, vol. 813, p. 590, 2006.

[220] S. Meir, C. Stephanos, T. H. Geballe, and J. Mannhart, "Highlyefficient thermoelectronic conversion of solar energy and heat into electric power," Journal of Renewable and Sustainable Energy, vol. 5, Article ID 043127, 2013.

[221] M. Terraneo, M. Peyrard, and G. Casati, "Controlling the energy flow in nonlinear lattices: a model for a thermal rectifier," Physical Review Letters, vol. 88, Article ID 094302, 4 pages, 2002.

[222] B. Li, L. Wang, and G. Casati, "Thermal diode: rectification of heat flux," Physical Review Letters, vol. 93, no. 18, Article ID 184301, 2004.

[223] C. W. Chang, D. Okawa, A. Majumdar, and A. Zettl, "Solid-state thermal rectifier," Science, vol. 314, no. 5802, pp. 1121-1124, 2006.

[224] R. Scheibner, M. König, D. Reuter et al., "Quantum dot as thermal rectifier," New Journal of Physics, vol. 10, Article ID 083016, 2008. 
[225] B. Li, L. Wang, and G. Casati, "Negative differential thermal resistance and thermal transistor," Applied Physics Letters, vol. 88, Article ID 143501, 2006.

[226] L. Wang and B. Li, "Thermal logic gates: computation with phonons," Physical Review Letters, vol. 99, no. 17, Article ID 177208, 4 pages, 2007.

[227] L. Wang and B. Li, "Thermal memory: a storage of phononic information," Physical Review Letters, vol. 101, no. 26, Article ID 267203, 4 pages, 2008.

[228] A. Khelif, B. Aoubiza, S. Mohammadi, A. Adibi, and V. Laude, "Complete band gaps in two-dimensional phononic crystal slabs,” Physical Review E, vol. 74, no. 4, Article ID 046610, 5 pages, 2006.

[229] I. U. Teudt, H. Maier, C.-P. Richter, and A. Kral, "Acoustic events and "optophonic" cochlear responses induced by pulsed nearinfrared LASER," IEEE Transactions on Biomedical Engineering, vol. 58, no. 6, pp. 1648-1655, 2011.

[230] A. Rosencwaig, Photoacoustics and Photoacoustic Spectroscopy, John Wiley \& Sons, 1980. 

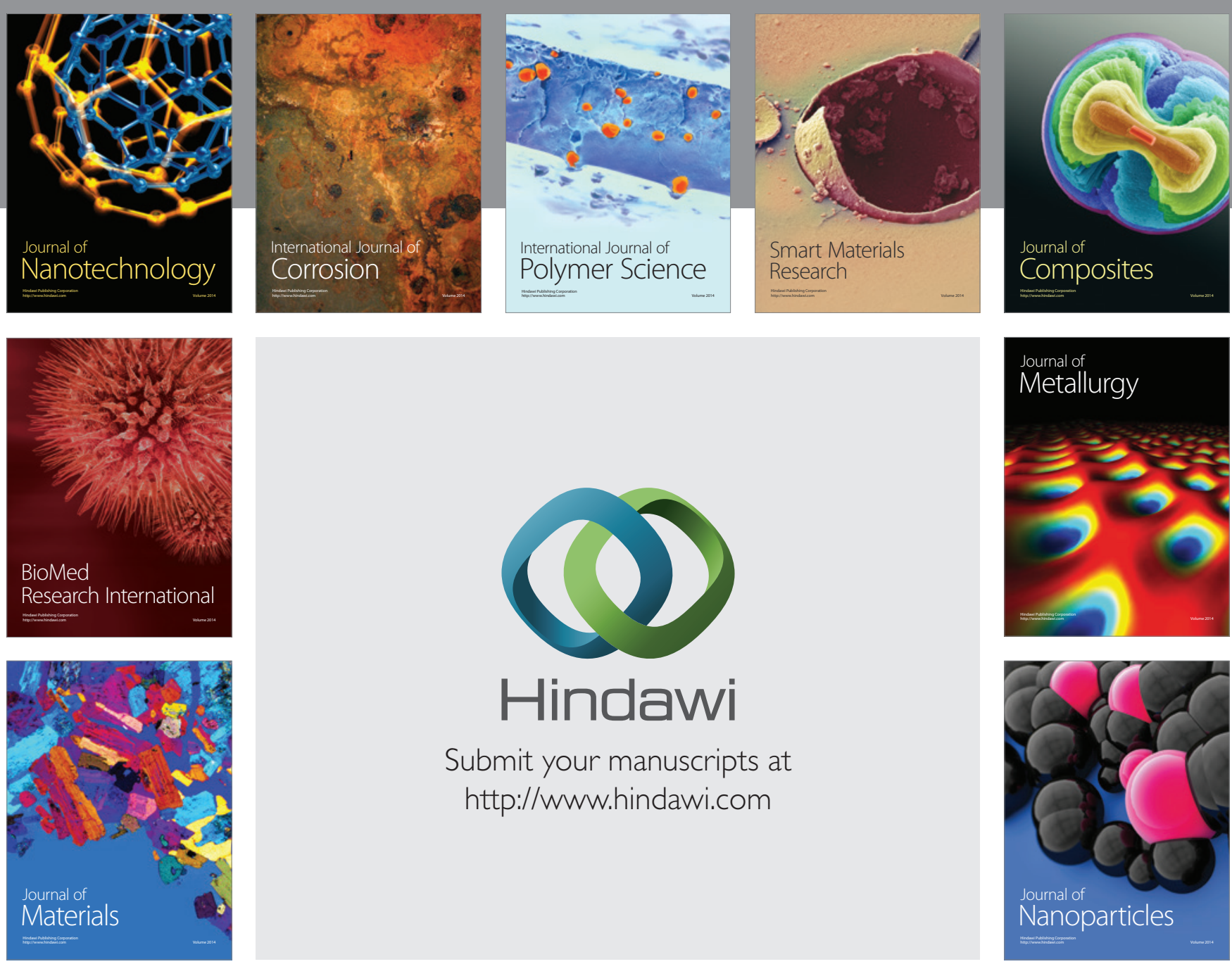

Submit your manuscripts at http://www.hindawi.com
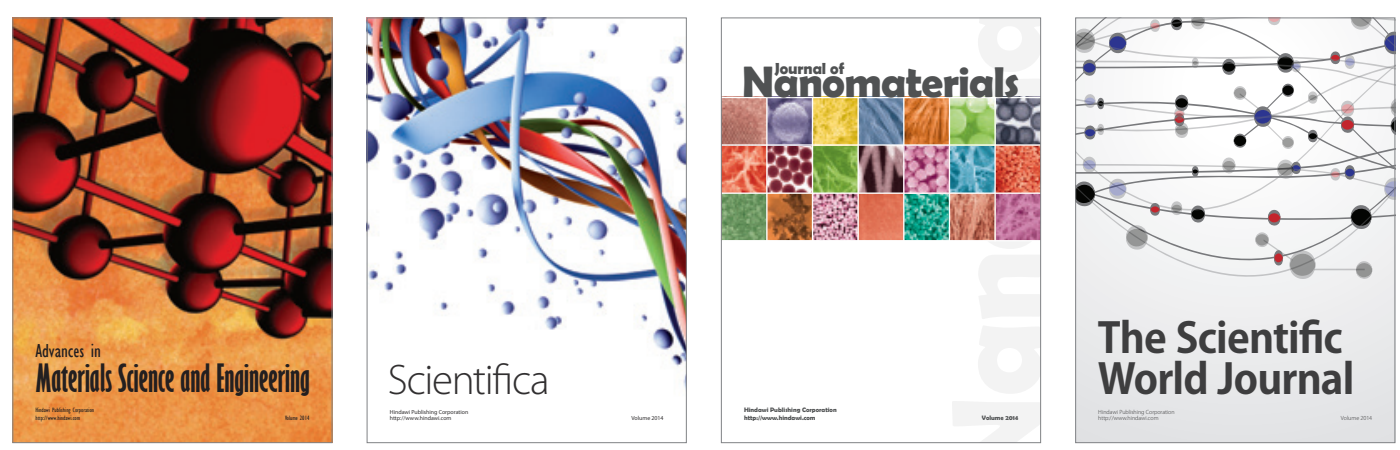

\section{The Scientific World Journal}
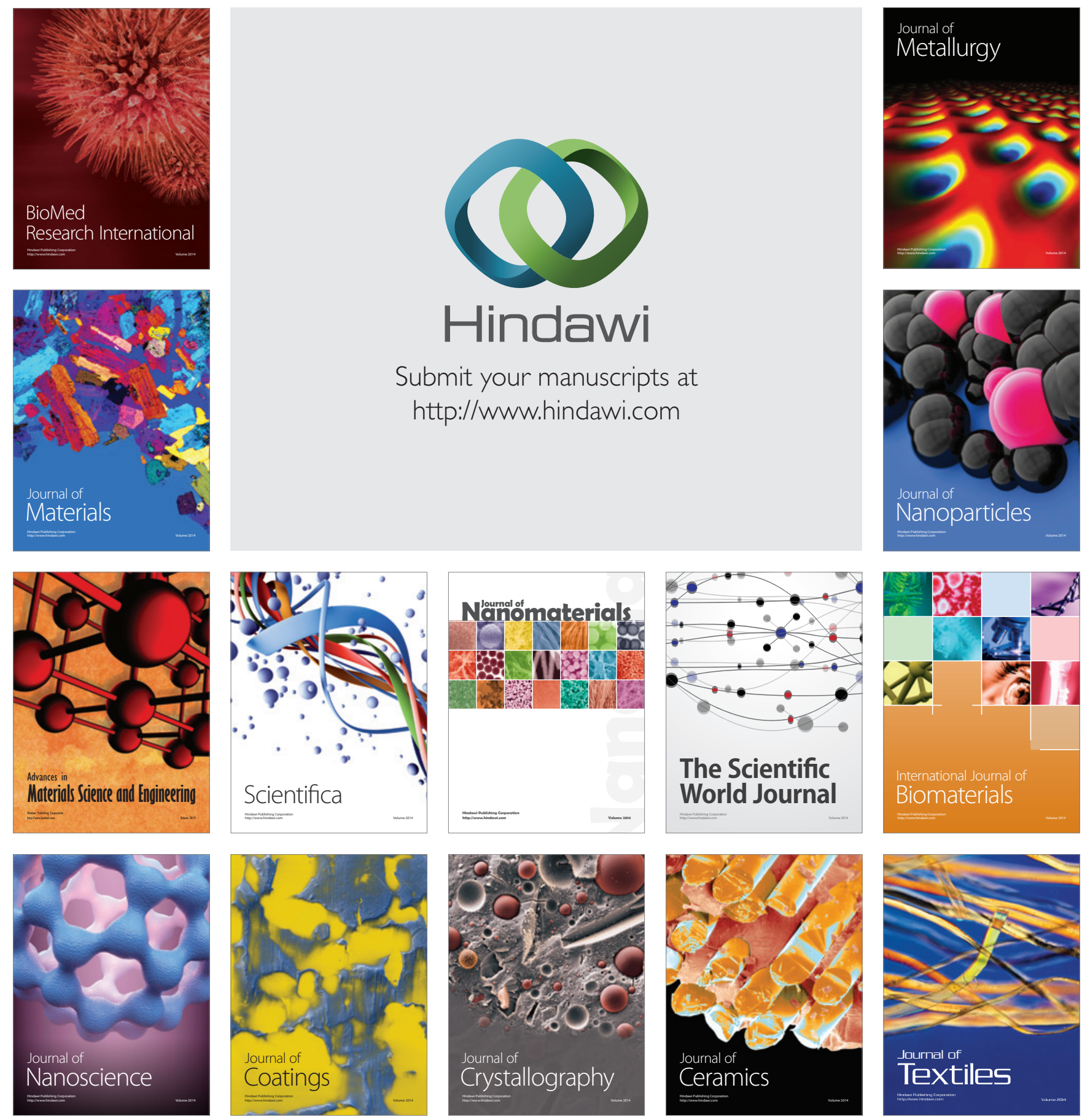